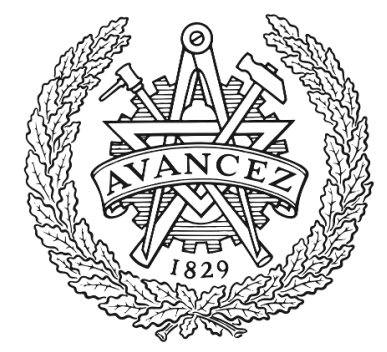

CHALMERS

UNIVERSITY OF TECHNOLOGY

\title{
Comparison of PANS and LES of the flow past a generic ship
}

Downloaded from: https://research.chalmers.se, 2023-04-26 14:53 UTC

Citation for the original published paper (version of record):

Zhang, J., Minelli, G., Rao, A. et al (2018). Comparison of PANS and LES of the flow past a generic ship. Ocean Engineering, 165: 221-236. http://dx.doi.org/10.1016/j.oceaneng.2018.07.023

N.B. When citing this work, cite the original published paper. 


\title{
Comparison of PANS and LES of the flow past a generic ship
}

\author{
Jie Zhang, ${ }^{\mathrm{a} *}$, Guglielmo Minelli ${ }^{\mathrm{a}}$, Anirudh Narayan Rao ${ }^{\mathrm{a}}$, Branislav Basara ${ }^{\mathrm{b}}$, Rickard Bensow ${ }^{\mathrm{a}}$, \\ Siniša Krajnović ${ }^{\mathrm{a}}$ \\ a Department of Mechanics and Maritime Sciences, Chalmers University of Technology, Gothenburg, 412 96, Sweden \\ ${ }^{\mathrm{b}}$ Advanced Simulation Technologies, AVL List GmbH, Hans-List-Platz 1, 8020, Graz, Austria
}

A R T I C L E I N F O

\section{Keywords:}

PANS

LES

Generic ship

Bi-stability

Separated flows

\begin{abstract}
A B S T R A C T
The prediction of flow past a generic ship has been performed using partially-averaged Navier-Stokes (PANS) turbulence model. The Reynolds number based on the width of the ship is $8 \times 10^{4}$. A detailed comparison with resolved large-eddy simulation (LES) solution and available experimental data is made for a better understanding of the capability of PANS in predicting the turbulent flow in the wake. The results show that PANS produces similar trends to those predicted by LES. However, a coarse grid resolution will lead PANS to act as unsteady Reynolds-averaged Navier-Stokes (URANS). Although the geometry is symmetric for the incoming flow at a zero yaw angle, bi-stable behaviour is observed in the wake, contributing to an asymmetric flow distribution on the heli-deck.
\end{abstract}

\section{Introduction}

In ship design, aerodynamic shapes have traditionally not been considered important, except in some special circumstances (e.g. in funnel design (Kulkarni et al., 2007; Park et al., 2017) to avoid smoke on deck of cruise ships or in helipad modification to improve operational safety of shipboard helicopters (Bardera and Meseguer, 2015)). Nevertheless, the ship external turbulent flow, characterized by massive separation, reattachment and shear layer evolution, has been attracting researchers to conduct investigations on the wake structures above deck (Syms, 2008; Herry, 2010; Forrest and Owen, 2010; Herry et al., 2011; Bardera and Meseguer, 2015; Kääriä et al., 2013; Rahimpour and Oshkai, 2016; Forrest et al., 2016). The prediction of this flow around a ship tends to be of great practical importance, due to the aerodynamic drag implications and large-scale separation forming in the wake.

In the last few years, the Reynolds-averaged Navier-Stokes (RANS) methods have been applied successfully in engineering applications, and appears to achieve an accurate prediction of attached flows as well as some cases with shallow separations, but it fails for massively separated flows. This is because the traditional RANS approach is modelling all the flow scales with one-point closures which cannot deal with bluff body flows where multi-point statistics plays an important role (Krajnović et al., 2012a). On the other hand, direct numerical simulation (DNS) resolves the entire range of spatial and temporal scales of turbulence without the need for any turbulence modelling. However, this method is so computationally demanding, that it is impractical for industrial turbulent flows. To solve the drawback of both methods, several implemented time-dependent simulation techniques, especially large-eddy simulation (LES), have been utilized in predicting the flow around different bluff vehicles, including trucks (Minelli et al., 2016), buses (Krajnović and Davidson, 2004) and trains (Östh and Krajnović, 2014). As to LES, its principal idea is to decrease the computational cost by modelling the small length scales which are the most computationally expensive to resolve. Based on this, the LES predictions show high accuracy, and good examples can be found in literature of ground vehicle prediction (Hemida and Krajnović, 2010; Krajnović, 2009) and bluff bodies (Krajnović, 2009; Hu et al., 2015). However, the Reynolds numbers in these simulations are much lower than the realistic ones. Despite of recent fast developments in high performance computing, it still remains very difficult and expensive for LES to be widely used in practical applications. Thus, there is a need for a numerical technique that is less dependent on the high mesh resolution requirements. As an alternative, several hybrid RANS/LES approaches (Spalart et al., 1997; Girimaji et al., 2003) that combine the advantages of RANS and LES methods have been proposed in recent years, aiming to reduce the computational expense. The switch between RANS and LES is often done in two ways, named zonal and bridging approaches, based on different treatments of the near-wall fine-scale flow and the far-field large-scale flow.

The detached-eddy simulation (DES), as a zonal approach firstly proposed by Spalart et al. (1997), is the hybrid method that attracts the greatest interest in the research community and industry. It has been

\footnotetext{
* Corresponding author.

E-mail address: zhjie@chalmers.se (J. Zhang).
} 
proved to be successful in several different bluff and streamlined body flows (Forrest and Owen, 2010; Hemida and Krajnović, 2009; Guilmineau et al., 2011; Zhang et al., 2016, 2018). The main characteristic of DES is that, the unsteady RANS (URANS) models are employed in the near-wall regions while the LES models are used in the regions away from the near-wall. Based on this, the method seems to be rather well understood, as it is easy to predict the behaviour of the method in different flow regions. However, an advantage sometimes can be a drawback, as there is a grey area between the RANS and LES, which is the definition of the interface between RANS and LES zones, especially for complex flows. In addition, as the near-wall flow is modeled using RANS, some important flow dynamics of the boundary layer can be lost. Therefore, the prediction of the flow is very sensitive to the computational grid.

The partially-averaged Navier-Stokes (PANS) is a bridging method originally proposed by Girimaji et al. (2003), and it represents an alternative to zonal methods. It enables a seamless transition from RANS (where all fluctuating scales are modeled) to DNS (where all fluctuating scales are resolved) depending on the dynamic control parameters $f_{k}$ (defined as the ratio of unresolved-to-total-kinetic energy) and $f_{\varepsilon}$ (defined as the ratio of unresolved-to-total-dissipation). With decreasing $f_{k}$, the modeled eddy viscosity decreases, leading to the resolution of more scales of coherent-structure motion and, consequently, improved predictions. Ideally, PANS will adapt to the existing computational grid by resolving the flow scales that can be resolved, and complementing with RANS where needed. This allows for flexibility in the method as RANS modelling can provide sufficient levels of Reynolds stresses when the grid is not adequate to resolve the turbulence. PANS has previously been used for several different bluff body flows, such as flows around cubes (Krajnović et al., 2012a, 2016), cylinders (Lakshmipathy and Girimaji, 2010; Jeong and Girimaji, 2010; Luo et al., 2014; Pereira et al., 2018) and simplified vehicles (Mirzaei et al., 2015; Han et al., 2013; Rao et al., 2018; Krajnović et al., 2012b). Most of these studies show that flow predictions of PANS are in good agreement with the experimental data, and often better than LES, when the mesh is relatively coarse. In this paper, PANS simulations of flow past a generic ship at Reynolds number $R e=8 \times 10^{4}$ have been performed and compared with LES. The main objective of the current work is not only to understand the flow physics around a ship, for example an interesting bistable flow has been observed and analysed in the wake of the ship (Syms, 2008; Herry, 2010; Herry et al., 2011), but also to assess the capability of PANS in predicting unsteady turbulent flows, and to conduct a detailed comparison with LES results and available experimental data.

\section{Methodology}

\subsection{Geometry}

The simplified ship model used in this study is shown in Fig. 1, and is constructed based on the model used in the wind tunnel tests described in Bardera and Meseguer (2015). It is a representative case for studying the flow around ship flight deck for experiments and numerical simulations. This model consists of the bow, superstructure, bridge, hangar and flight deck. The width of the ship (also known as the beam) is given by $W=0.15 \mathrm{~m}$, and is used as the characteristic dimension. All the quantities such as distances, shedding frequency are normalized by $W$ if not stated otherwise. All of edges in this model are sharp, resulting in fixed separations, typical of bluff body flows. The bow in the front of the ship is a triangular prism in plan view, and the heli-deck and the stern form a double backward-facing step at the rear.

\subsection{Governing equations}

LES and PANS were employed for the numerical study. The governing equations are shown as follows.

\subsubsection{Large-eddy simulation}

The governing LES equations are the spatially implicitly filtered Navier-Stokes equations, where the spatial filter is determined by the characteristic width $\Delta=\left(\Delta_{1} \Delta_{2} \Delta_{3}\right)^{\frac{1}{3}}$, and $\Delta_{i}$ is the computational cell size in the three coordinate directions.

The filtered momentum and continuity equations read:

$\frac{\partial \bar{u}_{i}}{\partial t}+\frac{\partial}{\partial x_{j}}\left(\bar{u}_{i} \bar{u}_{j}\right)=-\frac{1}{\rho} \frac{\partial \bar{p}}{\partial x_{i}}+\nu \frac{\partial^{2} \bar{u}_{i}}{\partial x_{j} \partial x_{j}}-\frac{\partial \tau_{i j}}{\partial x_{j}}$

and

$\frac{\partial \bar{u}_{i}}{\partial x_{i}}=0$

Here, $\bar{u}_{i}$ and $\bar{p}_{i}$ are the resolved filtered velocity and pressure, respectively. The influence of the small scales in Eq. (1) appears in the SGS stress tensor, $\tau_{i j}=\overline{u_{i} u_{j}}-\bar{u}_{i} \bar{u}_{j}$. These are unknown and must be modeled. The standard Smagorinsky model, described in Smagorinsky (1963), was used in the present work. The Smagorinsky model represents the anisotropic part of the SGS stress tensor, $\tau_{i j}$ as

$\tau_{i j}-\frac{1}{3} \delta_{i j} \tau_{k k}=-2 v_{s g s} \bar{S}_{i j}$

where $\bar{S}_{i j}$ is the resolved rate of strain,

$\bar{S}_{i j}=\frac{1}{2}\left(\frac{\partial \bar{u}_{i}}{\partial x_{j}}+\frac{\partial \bar{u}_{j}}{\partial x_{i}}\right)$

and $v_{s g}$ is the SGS viscosity defined as,

$v_{s g s}=\left(C_{s} f \Delta\right)^{2}|\bar{S}|$

where

$\bar{S}=\sqrt{\left(2 \overline{S_{i j}} \overline{S_{i j}}\right)}$

The Smagorinsky constant, $C_{S}=0.1$, previously used in bluff body LES (Krajnović, 2009), is used in the present work. The function $f$, in Eq. (5), is the Van Driest damping function,
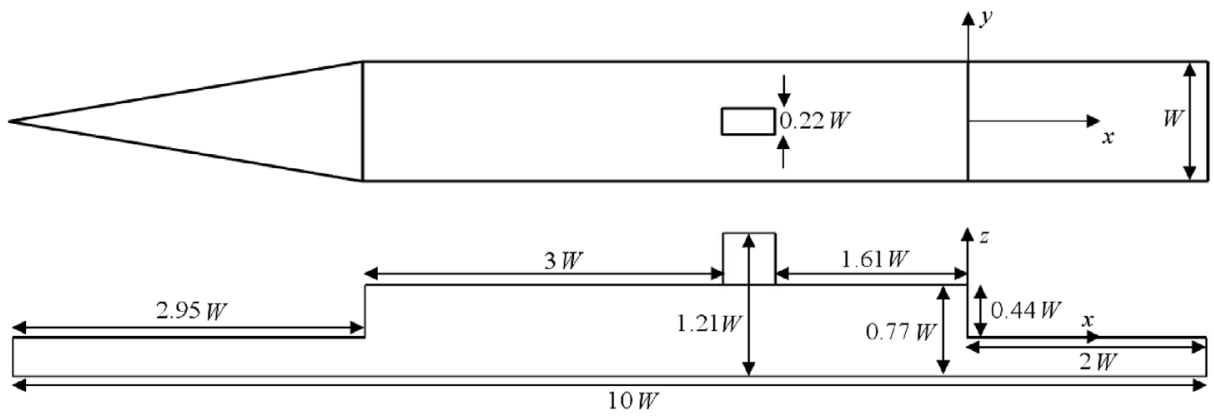

Fig. 1. Dimensions of the model in plan and left side views. 
$f=1-\exp \left(\frac{-n^{+}}{25}\right)$

where $n^{+}$is the wall normal distance in viscous units.

\subsubsection{Partially-averaged Navier-Stokes}

The governing equations is defined by the PANS model, (Girimaji, 2006; Girimaji et al., 2006).

$\frac{\partial U_{i}}{\partial x}=0$

$\frac{\partial U_{i}}{\partial t}+U_{j} \frac{\partial U_{i}}{\partial x_{j}}+\frac{\partial \tau\left(V_{i}, V_{j}\right)}{\partial x_{j}}=-\frac{1}{\rho} \frac{\partial p}{\partial x_{i}}+\nu \frac{\partial^{2} U_{i}}{\partial x_{j} \partial x_{j}}$

where $\tau\left(V_{i}, V_{j}\right)$ is the unresolved turbulent stress. This stress is modeled as:

$\tau\left(V_{i}, V_{j}\right)=-2 v_{u} S_{i j}+\frac{2}{3} k_{u} \delta_{i j}$.

Here $k_{u}$ is the unresolved kinetic energy, $S_{i j}=\frac{1}{2}\left(\partial U_{i} / \partial x_{j}+\partial U_{j} / \partial x_{i}\right)$ is the resolved stress tensor, and $v_{u}=C_{\mu} \zeta_{u} k_{u}^{2} / \varepsilon_{u}$ is the eddy viscosity of the unresolved scales where $\zeta=\overline{v_{u}^{2}} / k_{u}$ is the velocity scale ratio of the unresolved velocity scale $\overline{v_{u}^{2}}$ and $k_{u} \cdot \overline{v_{u}^{2}}$ refers to the normal fluctuating component of the velocity field to any no-slip boundary. At this stage three transport equations for $k_{u}-\varepsilon_{u}-\zeta_{u}$ and a Poisson equation for the elliptic relaxation function of the unresolved velocity scales are necessary to close the model. Thus the complete PANS $k-\varepsilon-\zeta-f$ model is given by the following set of equations:

$\frac{\partial k_{u}}{\partial t}+U_{j} \frac{\partial k_{u}}{\partial x_{j}}=P_{u}-\varepsilon_{u}+\frac{\partial}{\partial x_{j}}\left[\left(\nu+\frac{v_{u}}{\sigma_{k_{u}}}\right) \frac{\partial k_{u}}{\partial x_{j}}\right]$

$\frac{\partial \varepsilon_{u}}{\partial t}+U_{j} \frac{\partial \varepsilon_{u}}{\partial x_{j}}=C_{\varepsilon 1} P_{u} \frac{\varepsilon_{u}}{k_{u}}-C_{\varepsilon 2}^{*} \frac{\varepsilon_{u}^{2}}{k_{u}}+\frac{\partial}{\partial x_{j}}\left[\left(\nu+\frac{v_{u}}{\sigma_{\varepsilon_{u}}}\right) \frac{\partial \varepsilon_{u}}{\partial x_{j}}\right]$

$\frac{\partial \zeta_{u}}{\partial t}+U_{j} \frac{\partial \zeta_{u}}{\partial x_{j}}=f_{u}-\frac{\zeta_{u}}{k_{u}}\left(\varepsilon_{u}\left(1-f_{k}\right)-P_{u}\right)+\frac{\partial}{\partial x_{j}}\left[\left(\nu+\frac{\nu_{u}}{\sigma_{\zeta_{u}}}\right) \frac{\partial \zeta_{u}}{\partial x_{j}}\right]$

$L_{u}^{2} \nabla^{2} f_{u}-f_{u}=\frac{1}{T_{u}}\left(c_{1}+c_{2} \frac{P_{u}}{\varepsilon_{u}}\right)\left(\zeta_{u}-\frac{2}{3}\right)$.

As noted above, $v_{u}=C_{\mu} \zeta_{u} \frac{k_{u}^{2}}{\varepsilon_{u}}$ is the unresolved turbulent viscosity. $P_{u}=-\tau\left(V_{i}, V_{j}\right) \frac{\partial U_{i}}{\partial x_{j}}$ is the production of the unresolved turbulent kinetic energy and is closed by the Bousinnesq assumption, Eq. (10). The coefficients $C_{\varepsilon 2}^{*}$ and $C_{\varepsilon 1}$ are defined as:

$C_{\varepsilon 2}^{*}=C_{\varepsilon 1}+f_{k}\left(C_{\varepsilon 2}-C_{\varepsilon 1}\right)$

$C_{\varepsilon 1}=1.4\left(1+\frac{0.045}{\sqrt{\zeta_{u}}}\right)$.

Here, $\sigma_{k_{u}}=\sigma_{k} \frac{f_{k}^{2}}{f_{\varepsilon}}$ and $\sigma_{\varepsilon_{u}}=\sigma_{\varepsilon} \frac{f_{k}^{2}}{f_{\varepsilon}}$ are the counterpart of the unresolved kinetic energy and dissipation, respectively. The constants appearing in Eq. (11) are:

$C_{\mu}=0.22 ; \quad C_{\varepsilon 2}=1.9 ; \quad c_{1}=0.4 ; \quad c_{2}=0.65 ;$

$\sigma_{k}=1 ; \quad \sigma_{\varepsilon}=1.3 ; \quad \sigma_{\zeta_{u}}=1.2$.

$L_{u}$ and $T_{u}$ are the length and time scales defined by using the unresolved kinetic energy:

$T_{u}=\max \left[\frac{k_{u}}{\varepsilon}, C_{\tau}\left(\frac{v}{\varepsilon}\right)^{1 / 2}\right]$
$L_{u}=C_{L} \max \left[\frac{k_{u}^{3 / 2}}{\varepsilon}, C_{\eta}\left(\frac{\nu^{3}}{\varepsilon}\right)^{1 / 4}\right]$

where $C_{\tau}=6, C_{L}=0.36$ and $C_{\eta}=85$. A more detailed explanation of the construction of the equations is given in Basara et al. (2010, 2011). Parameters $f_{k}$ and $f_{\varepsilon}$ are the key factors that make the model act dynamically, and represent the ratios between resolved to total kinetic energy and dissipation, respectively. They can assume values between 1 and 0 according to the selected cut-off. The dynamic parameter was proposed as the ratio between the geometric averaged grid cell dimension, $\Delta=\left(\Delta_{x} \Delta_{y} \Delta_{z}\right)^{1 / 3}$, and the integral scale of turbulence, $\Lambda=\frac{\left(k_{u}+k_{\text {res }}\right)^{3 / 2}}{\varepsilon}$ (Girimaji and Abdol-Hamid, 2005):

$f_{k}(x, t)=\frac{1}{\sqrt{C_{\mu}}}\left(\frac{\Delta}{\Lambda}\right)^{2 / 3}$

\subsection{Numerical methods}

The simulations were carried out using a commercial finite volume CFD solver, AVL FIRE (AVL, 2014), based on the cell-centred finite volume approach. The convective terms in LES are approximated by a blend of $96 \%$ linear interpolation of second-order accuracy (central differencing scheme) and of $4 \%$ upwind differences of first order accuracy (upwind scheme). The diffusive terms containing viscous and sub-grid terms are approximated by a central differencing interpolation of second-order accuracy. According to the previous study on the influence of the numerical scheme in PANS (Krajnović et al., 2016), and the usage in the prediction of flow past Ahmed bodies in a squareback configuration (Mirzaei et al., 2015), a second-order AVL SMART Relaxed scheme (AVL, 2014; Pržulj and Basara, 2001) was used for convective terms in PANS simulations. However, as the choice of numerical requirements plays an important role in the simulations (Pereira et al., 2015, 2017; Krajnović et al., 2018), to evaluate the numerical errors of the simulations, a medium grid PANS simulation was performed using the same scheme in LES for the convective terms - central differencing scheme (CDS) as compared to AVL SMART Relaxed scheme (Appendix A). The time integration is done using the implicit second-order accurate three-time level scheme. To determine the pressure, Eq. (2) and Eq. (8) are converted into equations for the pressure correction. The SIMPLE algorithm (Patankar and Spalding, 1972) is used to update the pressure and velocity fields in order to satisfy the continuity equation.

\subsection{Computational domain and boundary conditions}

The ship model is mounted in a closed domain, as shown in Fig. 2. Similar to the experimental set-up of Bardera and Meseguer (2015), there is no clearance between the ship and the ground. Note that the free-surface effect of water is not involved in the present investigation. All dimensions are scaled by the width, $W$. The cross-sectional area of the domain is $10 \mathrm{~W} \times 10 \mathrm{~W}$, which gives a blockage ratio of $\simeq 1.21 \%$. The distance from the inlet to the front of the ship is $10 \mathrm{~W}$, and the distance from the stern to the outlet is $25 \mathrm{~W}$. The coordinate dimensions and velocities are denoted by $x$ and $u$ in the streamwise direction, $y$ and $v$ in the spanwise direction and $z$ and $w$ in the vertical direction, respectively. The coordinate origin is positioned in the symmetry plane, at the height of deck and at the end of the hangar, see Fig. 1.

For the simulations, the same boundary conditions were applied for both PANS and LES. A uniform velocity profile, constant in time was applied at the inlet. The Reynolds number is the same as for the wind tunnel test of Bardera and Meseguer (2015), $R e=8 \times 10^{4}$ based on the upstream velocity $U$ and the ship width $W$. A homogeneous Neumann boundary condition was applied $\left(\partial \bar{u}_{i} / \partial x_{i}=0\right)$ at the outlet. The surfaces of the body and the ground were treated as no-slip walls and the sides and roof are treated as symmetry walls $(\partial \bar{u} / \partial y=\partial \bar{w} / \partial y=\bar{v}=0$ on the sides and $\partial \bar{u} / \partial z=\partial \bar{v} / \partial z=\bar{w}=0$ on the top). The reference pressure with a value of $100,000 \mathrm{~Pa}$ is set at an undisturbed point, which is identical to the method used in wind tunnel tests.

\subsection{Computational grid}

Three different computational grids were used in this paper: coarse, medium and fine grids consisting of approximately 5.87, 9.93 and 21.25 million cells, respectively. The commercial grid generator software Ansys ICEM CFD was employed to create a hexahedral mesh 


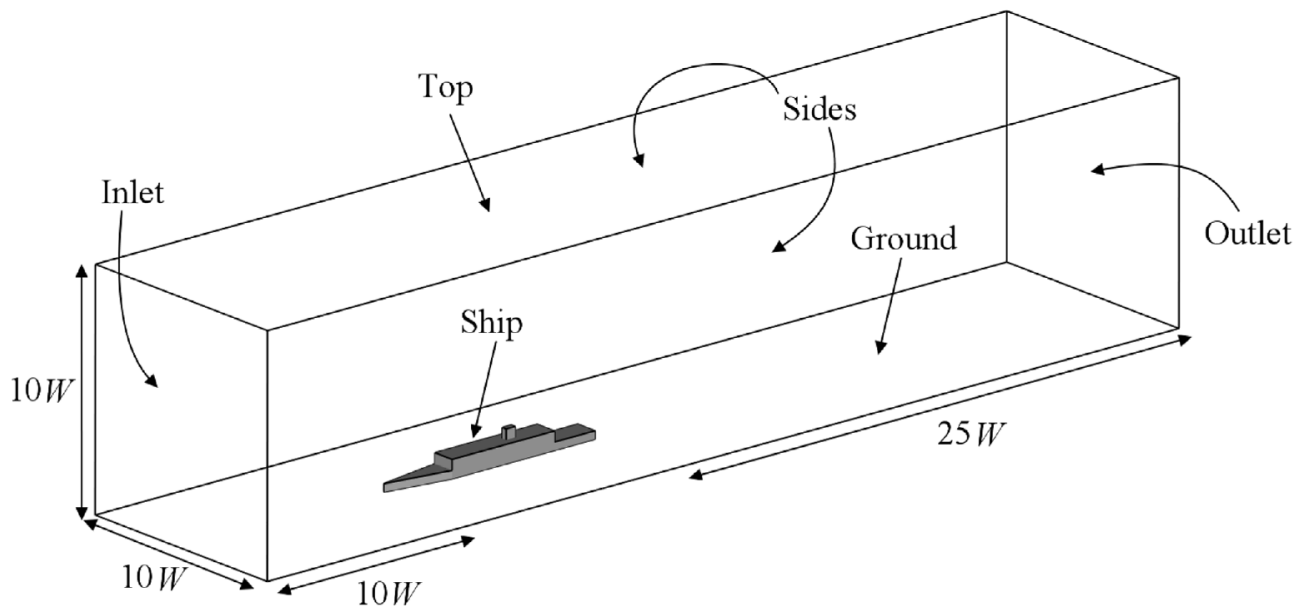

Fig. 2. Computational domain and boundary condition specification.
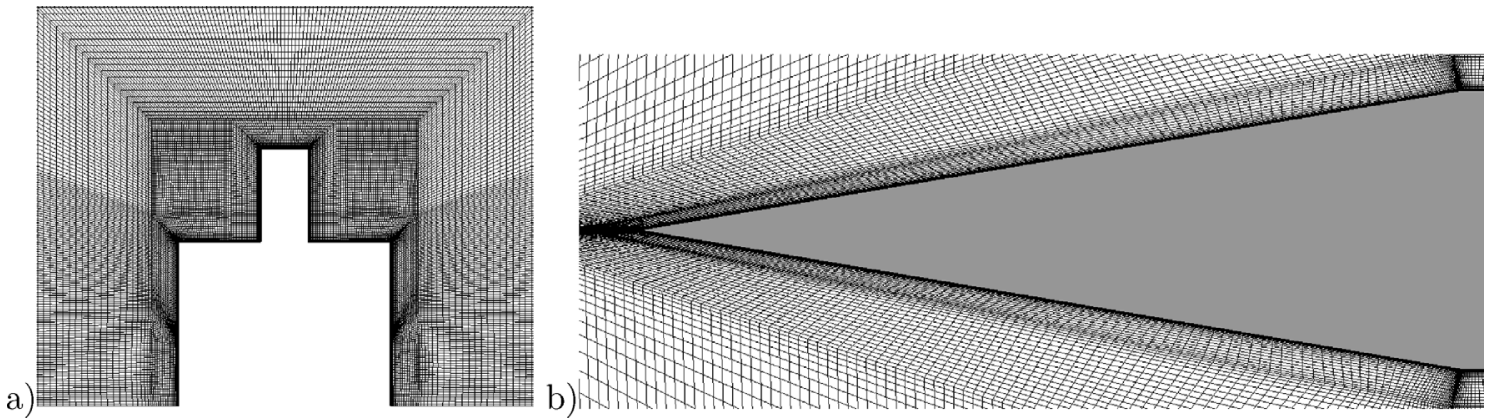

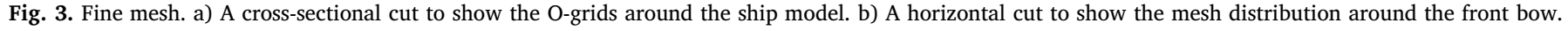

a)

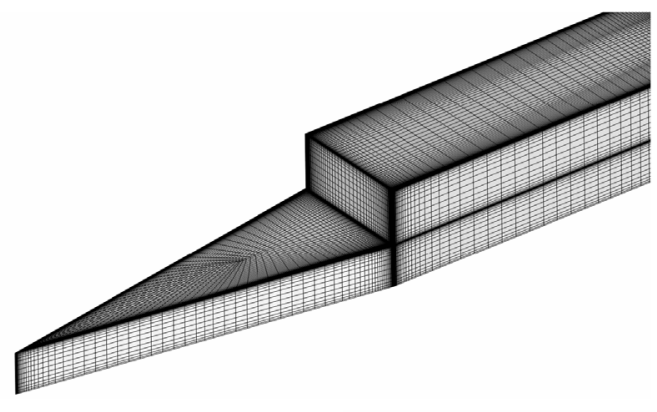

b)

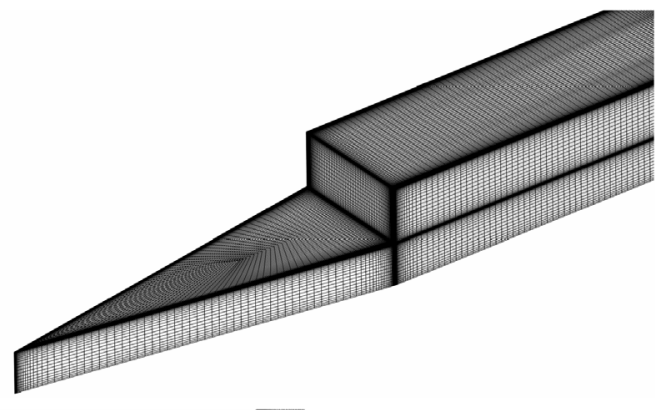

c)

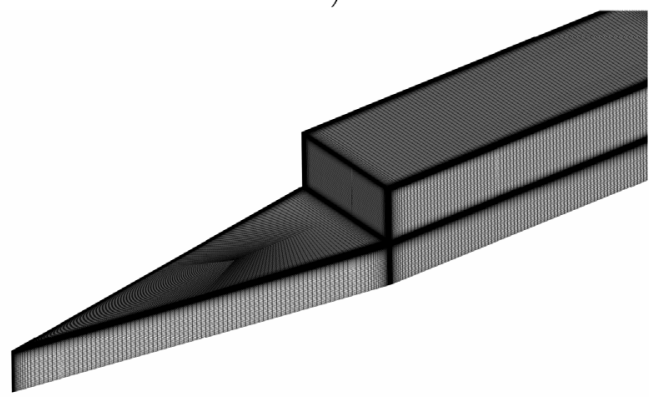

Fig. 4. Surface mesh distribution. a) Coarse mesh. b) Medium mesh. c) Fine mesh.

around the ship model. In order to capture the flow structures close to the ship accurately, the O-grid technique was used to concentrate most of the computational cells around the body, see Fig. 3a. The challenge on this grid is in dealing with the sharp bow of the ship, Fig. 3b. Fig. 4 shows the computational grid of the model's surface for the three meshes employed.

\subsection{Spatial and temporal resolution}

(Pope, 2001) suggests that a reliable LES grid should resolve $80 \%$ of the turbulence kinetic energy. According to (Piomelli and Chasnov, 1996), the first grid point in the wall normal direction must be located at $n^{+}<1$, where $n^{+}=\frac{u_{\tau} n}{\nu}$ with the friction velocity $u_{\tau}$. The resolution in the spanwise and streamwise directions must be $\Delta l^{+} \simeq 30-50$ and 
Table 1

Details of the computational grids.

\begin{tabular}{llll}
\hline Case & Fine grid & Medium grid & Coarse grid \\
\hline Number of cells & 21.25 million & 9.93 million & 5.87 million \\
\hline$n_{\text {mean }}^{+}$ & $<0.5$ & $<0.5$ & $<0.5$ \\
$\Delta l_{\text {max }}^{+}$ & $<50$ & $<100$ & $<150$ \\
$\Delta s_{\text {max }}^{+}$ & $<150$ & $<250$ & $<350$ \\
$\Delta l_{\text {mean }}^{+}$ & $<30$ & $<45$ & $<55$ \\
$\Delta s_{\text {mean }}^{+}$ & $<100$ & $<120$ & $<150$ \\
$C F L$ & $<1$ & $<1$ & $<1$ \\
& & &
\end{tabular}

$\Delta s^{+} \simeq 50-150$, respectively, in order to resolve the near-wall structures. Here $\Delta l^{+}=\frac{u_{\tau} \Delta l}{v}$ and $\Delta s^{+}=\frac{u_{\tau} \Delta s}{\nu}$. For the three grids the wall normal resolution on the ship model is $n^{+}<1$ with the size of the first cell in the normal direction being $0.00025 \mathrm{~W}$. The maximum values of $n^{+}$ are found on the front sharp edges of the bridge. The grid resolution of the three grids is described in Table 1 and visualized in Fig. 4. The physical time step for all cases, normalized by upstream velocity $U$ and ship width $W$, is $\Delta t^{*}=0.004$, giving a CFL number less than unity all over the domain. For each time step, the maximum number of iterations performed is set to 10 , while the minimum is 3 , which gives the normalized residual values of all quantities in the equations to less than $10^{-4}$. Additionally, to check iterative convergence at each time step, a PANS simulation on the medium mesh is conducted at the tolerance of $10^{-5}$ for the maximum normalized residual of all equations. For this geometry with sharp edges in the present work, a fully-developed flow is obtained before averaging the flow quantities. The simulations were carried out for $t^{*}=192$, which corresponds to approximately 4.3 flow passages through the domain, and averaging of the flow was started after $t^{*}=64$. The aerodynamic force coefficients of the ship are also monitored to guarantee the flow was fully-developed and converged.

\section{Results and discussion}

The PANS results using three different grids are presented in this section to understand the behaviour of the model in predicting complex flow structures. These results are also compared with the LES results of the current work and the available experimental data.

\subsection{Surface pressure}

The time-averaged pressure coefficients obtained by LES and PANS are plotted along three lines on the surface of the ship, as presented in Fig. 5. The lines are shown in Fig. 5a. Line $l_{y}$ starts on the front edge of the bow, and then goes along the symmetric line (at $y=0$ ) of the ship to the rear. Line $l_{z 1}$ lies in the $x-y$ plane at half of the bow of the ship from the ground, and line $l_{z 2}$ cuts the superstructure at half its height. Here, the pressure coefficient is defined as:

$C_{p}=\left(P-P_{r e f}\right) /\left(0.5 \rho U^{2}\right)$

where $P$ is the absolute pressure, $P_{\text {ref }}$ is the reference pressure of the undisturbed flow field, $\rho$ is the constant density of air which is 1.18858 $\mathrm{kg} / \mathrm{m}^{3}$.

In Fig. 5, it is observed that the results from the coarse to the fine grid simulations give a very similar prediction on the bow, funnel and hangar faces of the ship. However, larger deviations are present along the separation regions on the bridge, funnel and rear faces. The $C_{p}$ along line $l_{y}$ (Fig. 5b) for the LES, shows a higher pressure on the front edge of the bow, while PANS, using a coarse grid, clearly fails to predict the pressure in the front of the bridge where the flow separation is defined by the geometry rather than by the adverse pressure gradient. The reason why PANS fails is related to poor resolution of the near-wall as refining the grid gives better solution. This is also found in the study of surface mounted cube flow (Krajnović et al., 2016). The PANS technique on the other hand uses more turbulence modelling when the grid is coarse, resulting in underestimating the flow characteristics. However, with grid refinement, more turbulence is resolved, and less turbulence modelling is used, giving better prediction of the separation region. The results from the coarse to fine grid simulations show high consistency, except for the pressure on the transition faces from the bow to sides and on the rear faces, as shown Fig. $5 \mathrm{~d}$.

\subsection{Aerodynamic forces}

Table 2 shows the time-averaged and the standard deviation $(\sigma)$ values of the aerodynamic force coefficients for all the cases studied in the present work. The time histories of the side forces and the corresponding power spectral density (PSD) are shown in Appendix B. Here the aerodynamic coefficients are defined as

$\left\langle C_{x}\right\rangle=\left\langle F_{x}\right\rangle /\left(0.5 \rho U^{2} A\right),\left\langle C_{y}\right\rangle=\left\langle F_{y}\right\rangle /\left(0.5 \rho U^{2} A\right),\left\langle C_{z}\right\rangle=\left\langle F_{z}\right\rangle /\left(0.5 \rho U^{2} A\right)$

where $\left\langle F_{x}\right\rangle,\left\langle F_{y}\right\rangle$ and $\left\langle F_{z}\right\rangle$ are the drag force, side force and lift force, respectively. Here, $A$ is the frontal cross-sectional area without considering the funnel. The $\left\langle C_{x}\right\rangle$ is around 0.70 for all cases, while the $\left\langle C_{z}\right\rangle$ is higher in LES than in PANS. The $\left\langle C_{x}\right\rangle$ and $\left\langle C_{z}\right\rangle$ experienced by the ship model using the medium mesh show a difference of less than $2.2 \%$. For the coarse mesh, the differences are still within $4 \%$ as compared to the fine mesh. The standard deviation values of the force coefficients on the same grid resolution are lower in LES than in PANS. When the tolerance for the maximum normalized residual of all equations reduces from $10^{-4}$ to $10^{-5}$, the $\left\langle C_{x}\right\rangle$ and $\left\langle C_{z}\right\rangle$ change approximately by $-0.30 \%$ and $0.66 \%$, respectively, compared to those obtained using PANS on the same grid. This indicates a tolerance of $10^{-4}$ for the maximum value of the normalized residual of all equations is sufficient for the present simulations.

\subsection{Analysis of ship flow}

In this section, the flow around the ship for all cases is shown by the instantaneous flow structures, time-averaged velocity profiles, and streamlines and unresolved turbulence kinetic energy.

\subsubsection{Instantaneous flow structures}

Fig. 6 visualizes instantaneous flow structures around the ship from a front view, predicted by LES and PANS simulations. All results are shown for three meshes: coarse, medium and fine meshes. The structures are presented by iso-surfaces of positive values of the second invariant of the velocity gradient tensor, $Q$-criterion. An iso-surface with positive $Q$ reveals locations in the flow where the strength of the rotation overcomes the strain, thus indicating vortical structures (Hunt et al., 1988). For an incompressible flow, the $Q$ can be defined as:

$Q=-\frac{1}{2}\left(\frac{\partial u_{i}}{\partial x_{j}} \frac{\partial u_{j}}{\partial x_{i}}\right)=-\frac{1}{2}\left(\bar{S}_{i j} \bar{S}_{i j}-\bar{\Omega}_{i j} \bar{\Omega}_{i j}\right)$.

where $\bar{S}_{i j}$ and $\bar{\Omega}_{i j}$ are the symmetric and anti-symmetric parts of the resolved velocity gradient tensor.

Fig. 6 indicates that the LES resolves more flow structures due to the well-resolved grid resolution around the ship body, while the PANS, except for the coarse grid, is clearly successful in resolving the large unsteady flow structures. All six cases can predict the separated flow region on the top of the bridge and in the wake. For the PANS simulation on the coarse mesh, only the large-scale flow structures are resolved. The flows predicted by PANS become similar to these found in LES by increasing the grid resolution. 


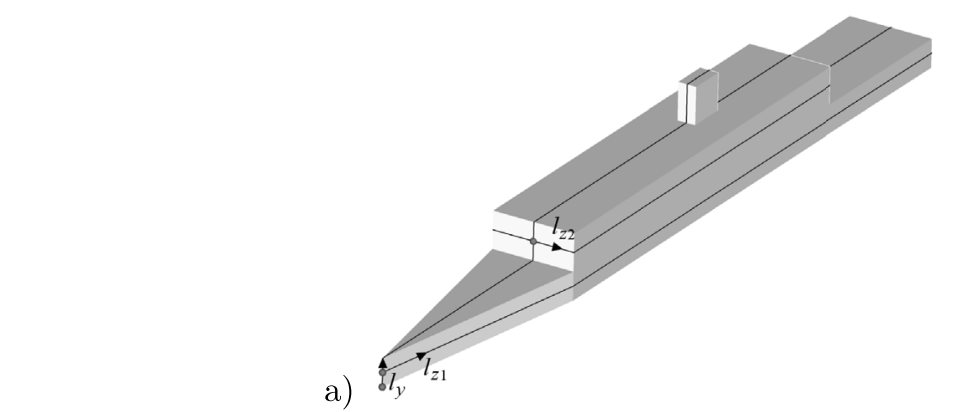

b)
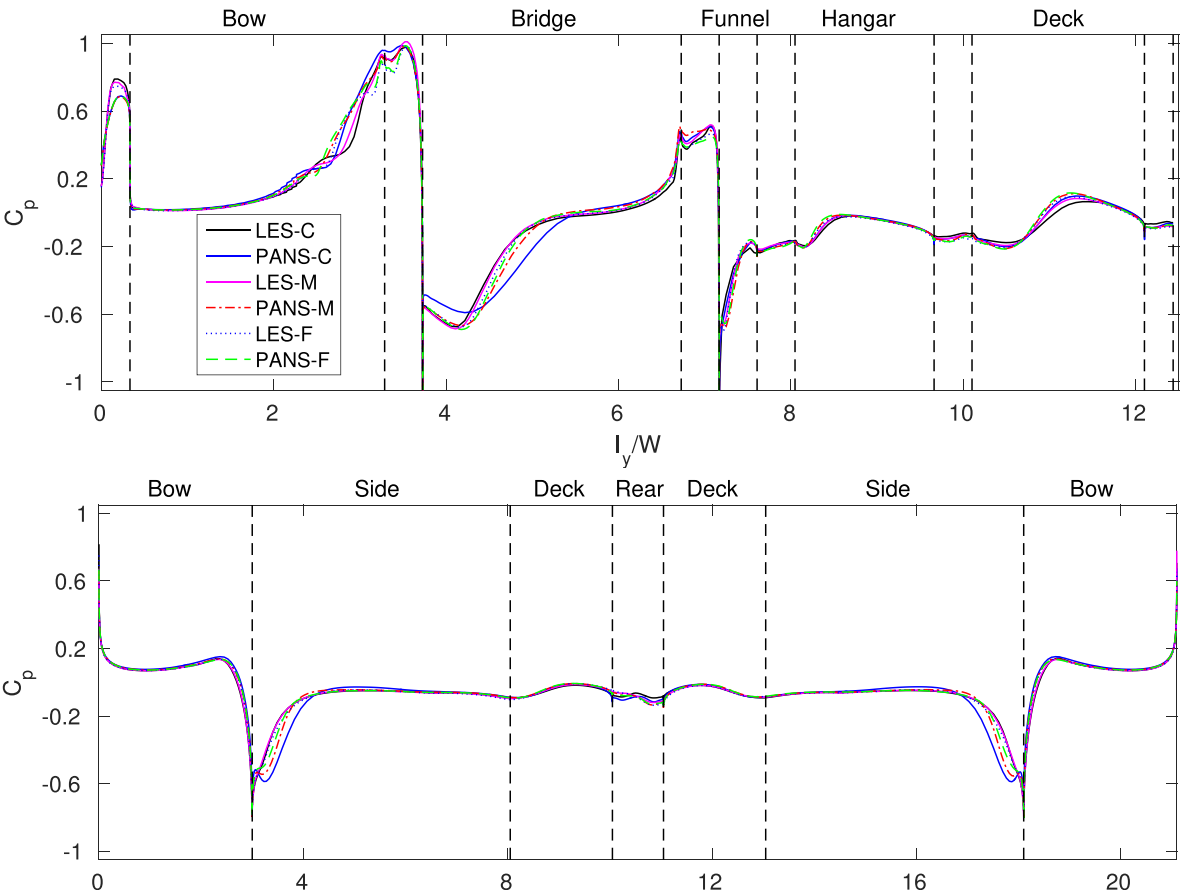

c)

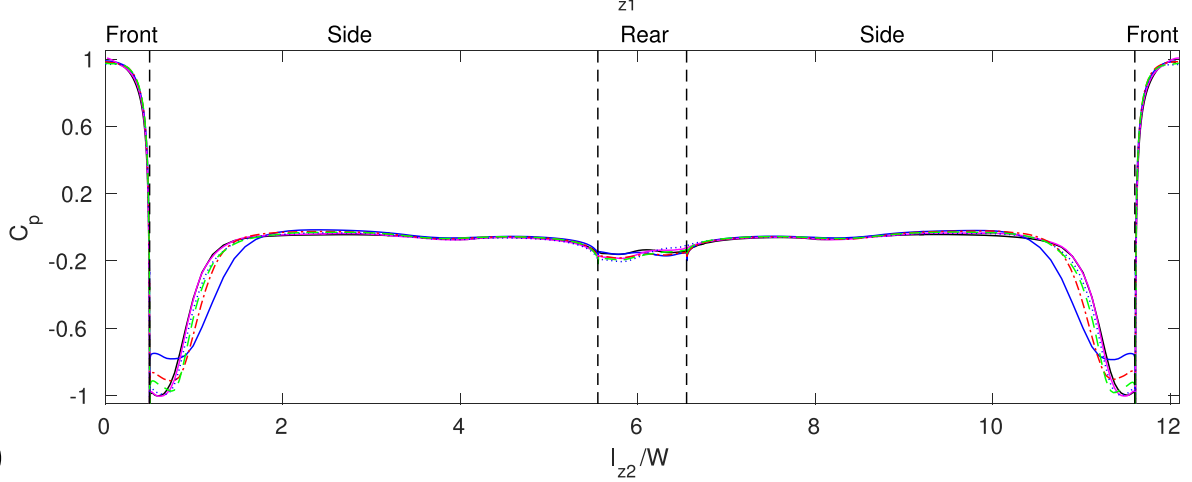

Fig. 5. $C_{p}$ plotted along four lines on the surface of the ship comparing with values from three grid simulations: a) Location of profiles. b) $C_{p}$ along line $l_{y}$. c) $C_{p}$ along line $l_{z 1}$. d) $C_{p}$ along line $l_{z 2}$. Legends: C: coarse mesh; M: medium mesh and F: fine mesh. These labels are also used in Figs. 7, 8 and 9.

Table 2

Aerodynamic forces and standard deviation $(\sigma)$ values.

\begin{tabular}{|c|c|c|c|c|c|c|c|c|}
\hline Case & Cells (million) & Residual & $\left\langle C_{x}\right\rangle$ & $\sigma\left(C_{x}\right)$ & $\left\langle C_{y}\right\rangle$ & $\sigma\left(C_{y}\right)$ & $\left\langle C_{z}\right\rangle$ & $\sigma\left(C_{z}\right)$ \\
\hline LES & 5.87 & $10^{-4}$ & 0.7114 & 0.0189 & -0.0014 & 0.0383 & 0.7892 & 0.0226 \\
\hline LES & 9.93 & $10^{-4}$ & 0.7177 & 0.0201 & 0.0034 & 0.0396 & 0.7784 & 0.0198 \\
\hline PANS & 5.87 & $10^{-4}$ & 0.7164 & 0.0253 & 0.0037 & 0.0656 & 0.7606 & 0.0375 \\
\hline PANS & 9.93 & $10^{-4}$ & 0.7058 & 0.0252 & 0.0037 & 0.0534 & 0.7411 & 0.0310 \\
\hline PANS & 21.25 & $10^{-4}$ & 0.6909 & 0.0194 & -0.0036 & 0.0459 & 0.7319 & 0.0275 \\
\hline
\end{tabular}


a)

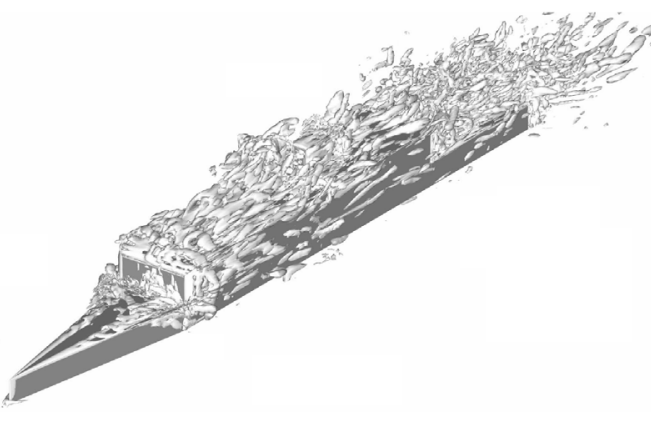

b)

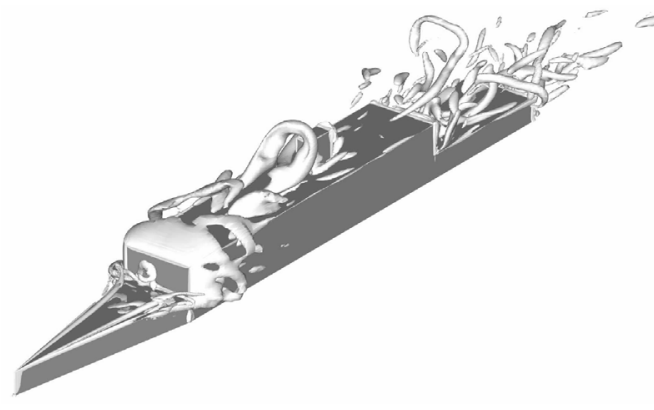

d)

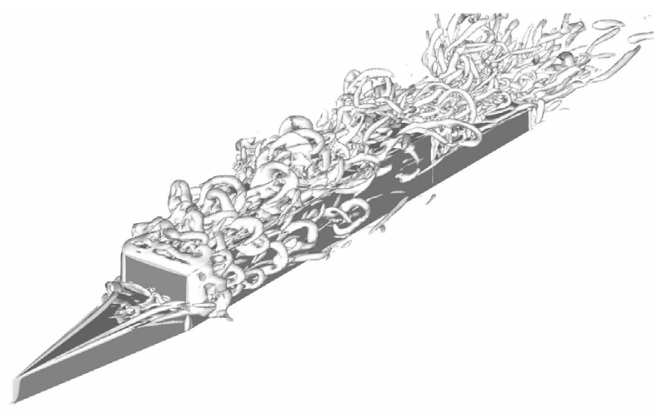

c)

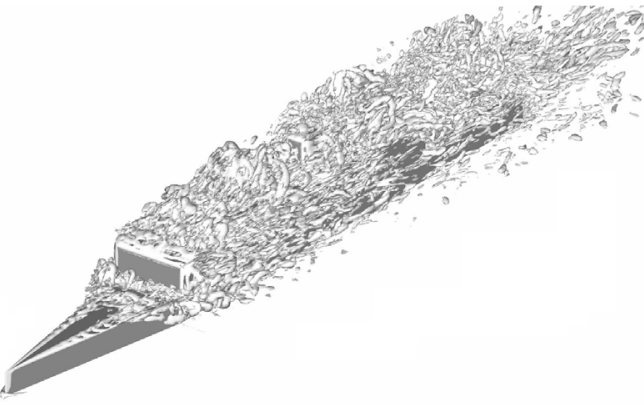

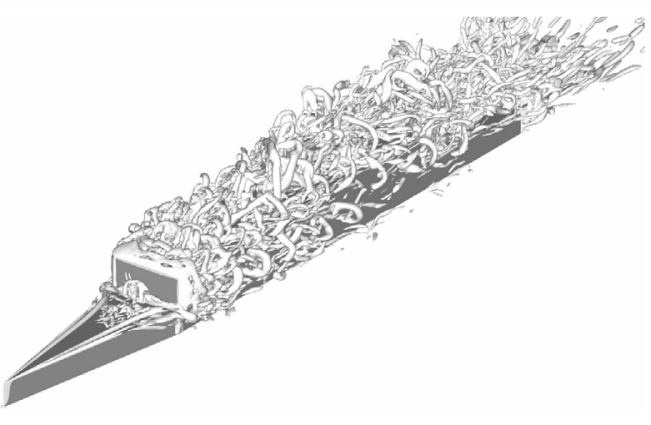

e)

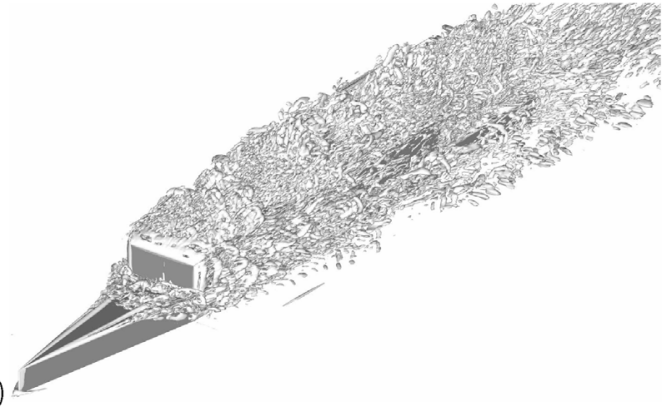

f)

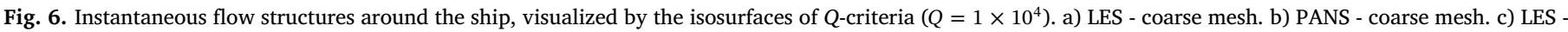
medium mesh. d) PANS - medium mesh. e) LES - fine mesh. f) PANS - fine mesh. Flow is from bottom left to top right in these images.

\subsubsection{Velocity profiles}

Fig. 7 shows the time-averaged streamwise velocity component along six different lines from the bow to the flight deck in the symmetry plane, predicted by LES and PANS on the three grids. The location of the lines are presented in Fig. 7a. The general observation is that PANS simulations produce similar trends to those predicted by LES. A large discrepancy is observed between the coarse grid PANS and the other cases along the second line, while a reversed flow and negative velocity is presented in PANS on a coarse mesh. Along the third line, the velocities predicted by PANS are larger at the height below $0.4 \mathrm{~W}$. However, when the height is higher than $0.4 \mathrm{~W}$, the velocity predicted by the coarse grid PANS shows a smaller value and the one obtained by the coarse grid LES gives a larger one. Note that for the velocity profiles along lines No. 4 to No. 6 , a considerably better prediction is obtained by the medium grid PANS, as compared to the results from the coarse and fine grid PANS and medium grid LES. The coarse grid LES gives the worst prediction below the height of the hangar, especially for the velocities along lines No. 5 and No. 6. Along line No. 4, reversed velocities are all predicted by PANS and LES, while the velocity for PANS in the same grid is larger due to large downwash coming from the superstructure. There are regions of reversed flow observed in the coarse and medium grid LES and coarse grid PANS along the fifth line, which shows an incorrect prediction, as no reversed velocity is observed in the fine grid LES.

The time-averaged streamwise velocity component along four lines across the flight deck at hangar height $(z=0.44 W)$ is presented in Fig. 8, obtained using PANS and LES. The location of the lines are presented in Fig. 8a. From the sides towards the centreline, a streamwise velocity reduction can be clearly found along lines No. 1 to No. 4, which indicates that the flow is affected by the hangar and superstructure of the ship. The corresponding downwash can be observed (see Fig. 11), showing that the flow coming from the hangar top is impacting directly on the deck. LES simulations give lower velocities at the peak positions than those predicted by PANS on the same grid. For the coarse grid PANS and LES, from $y=-0.5 \mathrm{~W}$ to $y=0.5 \mathrm{~W}$, large differences are shown without the obvious asymmetry, compared to the medium and fine grid PANS and LES. It is very interesting to note that the velocities are distributed asymmetrically when the fine grid is used, as shown in these velocity contours in Fig. 15d-g, especially when the streamlines are plotted. Although the geometry is symmetric with a zero degree yaw angle, the time-averaged streamwise velocity is far from being symmetric. This asymmetry is already mentioned by Syms on the SFS2 (Simple Frigate Shape 2) (Syms, 2008), and a similar feature is also described in Herry (2010) and Herry et al. (2011).

Fig. 9 shows the comparison of time-averaged streamwise velocities obtained by numerical simulations using PANS/LES $\left(R e=8 \times 10^{4}\right)$ and previous numerical simulations of Forrest and Owen (2010) $(R e=$ $\left.6.58 \times 10^{5}\right)$ and Syms $(2008)\left(R e=5.59 \times 10^{5}\right)$, and experiments of the Aerodynamics Laboratory (AL) of the National Research Council, Canada (NRC) $\left(R e=5.59 \times 10^{5}\right)$. Although the Reynolds numbers are not the same, the comparison is qualitative to show the similar behaviour of the flow instability. According to the wind tunnel tests performed in the Aerodynamics Laboratory (AL) of the National Research Council, Canada (NRC) and numerical simulations conducted by Syms (2008), a mean asymmetric flow at zero degree yaw is observed. Syms (2008) supposes that the flow might lock into one side of the bow as the 
a)
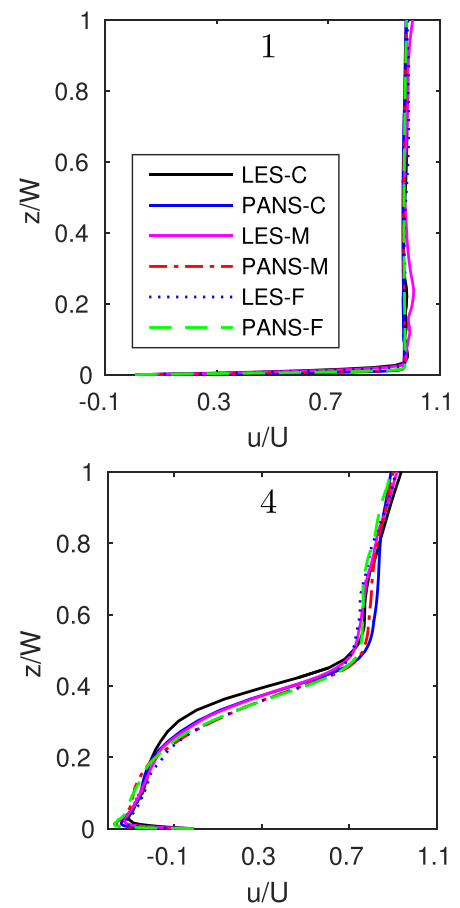
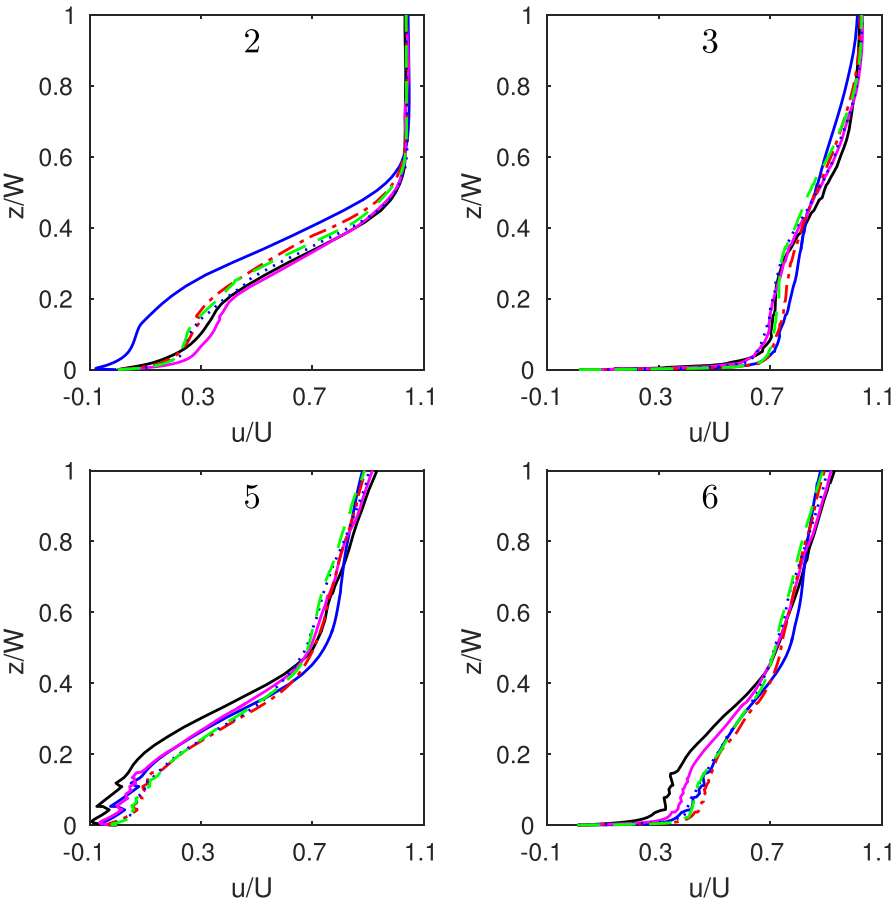

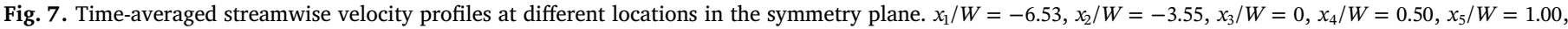
$x_{6} / W=1.50$. Flow is from left to right in these images.

bow itself is fairly long and narrow. Consequently, a fully-symmetric flow would become an unstable one. Forrest and Owen (2010) also computed the flow around the same ship model using DES, however, this asymmetric velocity profile is not clearly evident in their work. In the present work, the velocity profiles predicted by the medium and fine grid PANS and LES indicate this asymmetric phenomenon again. The location of the funnel in the current study is farther to the base of the hangar in the streamwise direction (see the model in Fig. 1 and the geometry in Syms (2008)), and the distance downstream from the start of the boundary layer behind the funnel is longer; lower velocities are thus expected in the wake behind the hangar and funnel. The trends shown in the wind tunnel data are consistent with the CFD results obtained by the fine grid PANS and LES.

\subsubsection{Streamlines}

In Section 3.3.2, a large difference is observed between the coarse grid PANS and the rest simulations along the second line in Fig. 7a. To find out what causes such large discrepancy, the time-averaged streamwise velocity contours and streamlines in the front symmetry plane predicted by LES and PANS are shown in Fig. 10. PANS using a coarse mesh clearly fails to predict the separation bubble in the front of the superstructure, Fig. 10c. A coarse grid resolution leads to rapid increase of unresolved eddy viscosity as well as increasing unresolved turbulent kinetic energy (Fig. 13b), and the production of the unresolved turbulent kinetic energy is computed using eddy viscosity modelling, a larger $P_{k u}$ will be produced. Thereby, PANS will act as URANS, resulting in an incorrect flow prediction. Additionally, LES results show very similar velocity contours and streamlines, although these slightly change with grid refinement.

To determine the accuracy of the flow prediction of the two turbulence modelling approaches, the flow structures in the wake are compared. Fig. 11 shows the time-averaged streamwise velocity contours and streamlines in the symmetry plane behind the body, predicted by LES and PANS on the fine mesh. Generally, the downwash from the hangar in the wake approximates to the flow behind a 3D double backward facing step. Behind the first step, it separates from the hangar roof and reattaches on the deck, forming a large recirculation bubble and a secondary (smaller) recirculation bubble, characterized by low velocity, at the corner of the step, adjacent to the vertical face. Similar flow characteristics can be found behind the second step.

According to these qualitative analysis, it is still hard to know how accurate the numerical methods are. Therefore, the reattaching length $X_{R i(i=1,2)}$ and the location of the center $\left(X_{V}, Z_{V}\right)$ of the larger vortices for each of the cases investigated are shown in Table 3. Additionally, the reattachment length can be obtained through the method illustrated in Fig. 12, since the reattachment point is where wall shear stress is zero. The wall shear stress can be calculated as follows: $\tau=\mu\left(\frac{\partial u}{\partial z}\right)_{z=0}$. Here, $\mu$ is the dynamic viscosity, $u$ is the flow velocity parallel to the wall and $z$ is the distance to the wall. According to the formula, if $\tau=0, \partial u$ that can be rewritten as $\partial u=\Delta u=u_{1}-u_{0}$ should be 0 , as $z>0$. Having the velocity on the wall $u_{0}$ is $0, u_{1}$ at the location $z$ should also be zero. This point can therefore be considered as the reattaching point. Here, two lines are used to extract the velocity data. The upper line corresponds to the profile shown in the wind tunnel tests (Bardera and Meseguer, 2015), while the lower line attempts to predict the actual reattachment length. The medium grid PANS shows a good prediction in terms of the reattachment length, compared to the fine grid LES and the experiments. However, the locations of the center of the larger vortices show small differences in PANS and LES, while the LES results seem to be 
a)

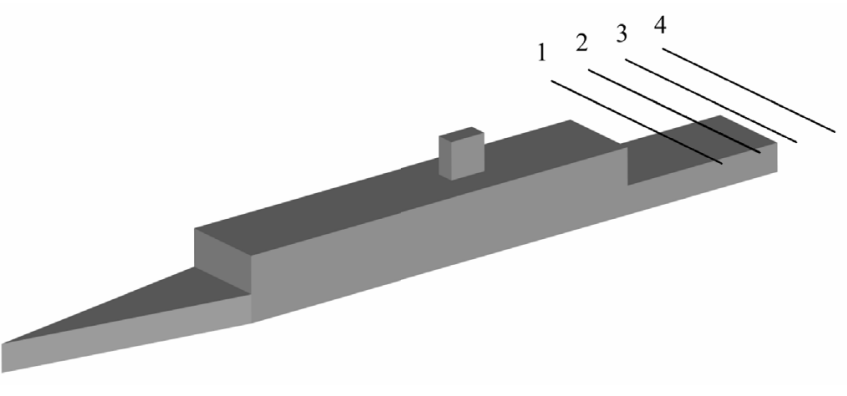

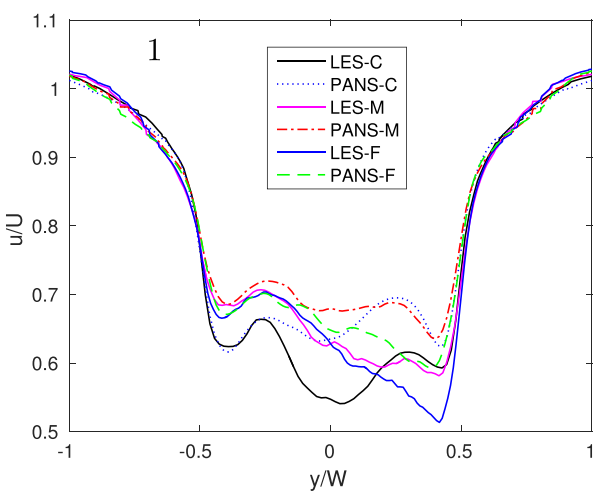
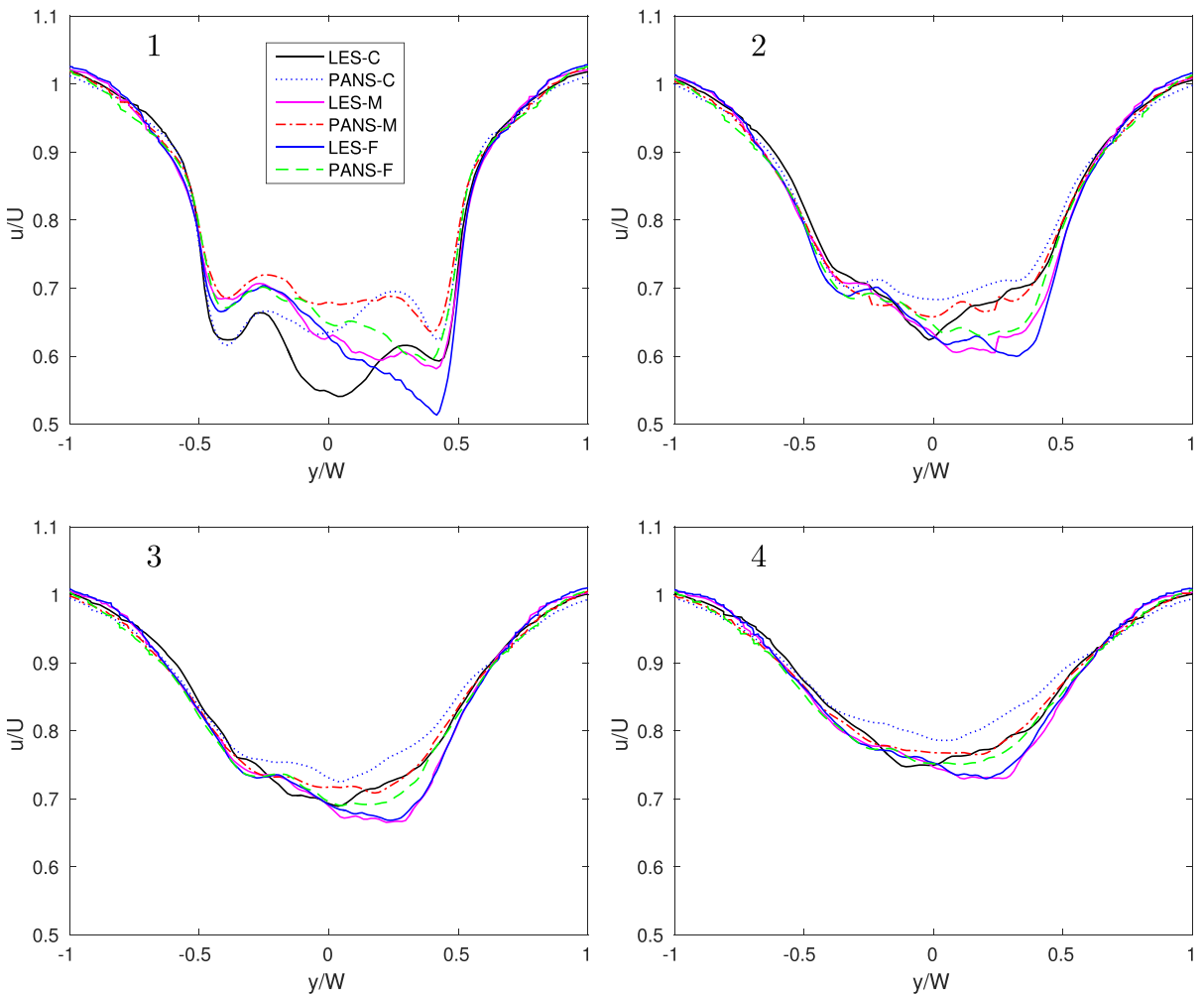

Fig. 8. Time-averaged streamwise velocity profiles at different locations in the $z=0.44 W$ plane. $x_{1} / W=0.50, x_{2} / W=1.00, x_{3} / W=1.50, x_{4} / W=2.00$.

more sensitive to the grid resolution. On the fine grid, the prediction of the flow and the vortex centres obtained in PANS are in good agreement with LES.

\subsection{4. $f_{k}$ in PANS and unresolved turbulence kinetic energy}

Fig. 13 compares unresolved turbulence kinetic energy $k_{u}$, obtained by PANS and LES with three different grids.
According to the governing equations in Section $2, k_{u}$ in PANS is derived from the transport equation, while in LES it is computed from the expression for the Smagorinsky model as $k_{u}=k_{\text {sgs }}=\left(C_{s} \Delta|\bar{S}|\right)^{2} / 0.3$ (Krajnović et al., 2012a). As PANS is a hybrid RANS/LES approach, the underlying RANS model will model a larger fraction of the turbulence kinetic energy than the subgrid-scale model in a LES. Therefore, the unresolved turbulent kinetic energy $k_{u}$ in PANS is expected to be larger

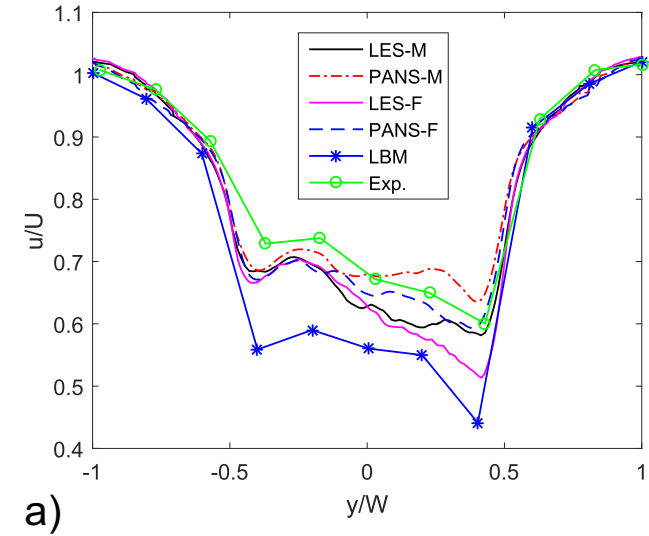

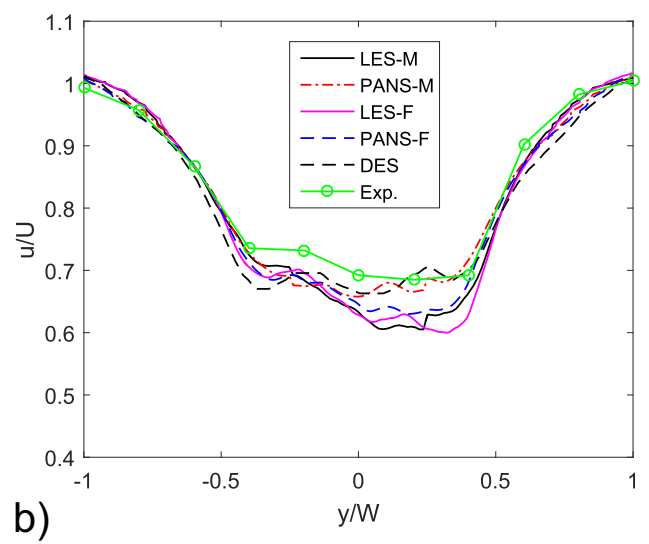

Fig. 9. Comparison of time-averaged streamwise velocity profiles along two lateral lines downstream of the hangar using numerical and experimental methods: Lattice-Boltzmann method (LBM) of Syms (2008), DES of Forrest and Owen (2010) and experiment (Exp.) of AL of NRC. a) At $25 \%$ length of the flight deck. b) At $50 \%$ length of the flight deck. 
a)

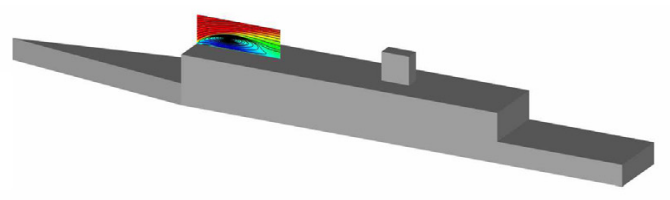

b)

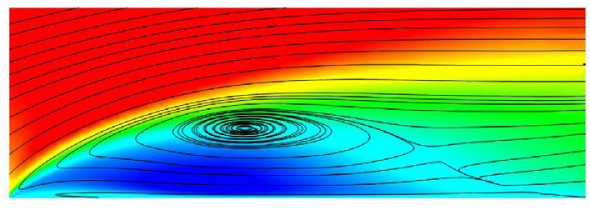

d)

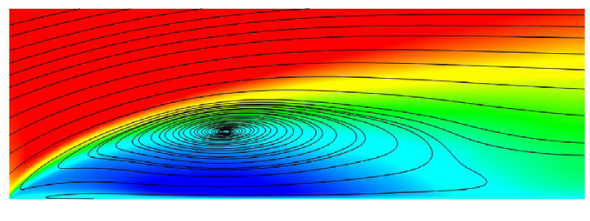

f)

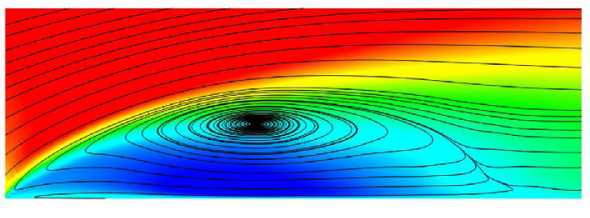

c)

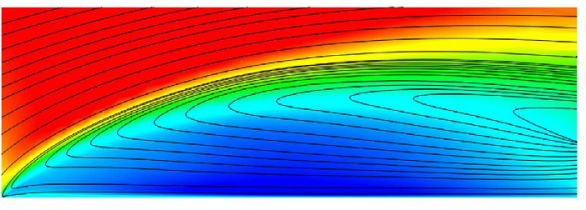

e)

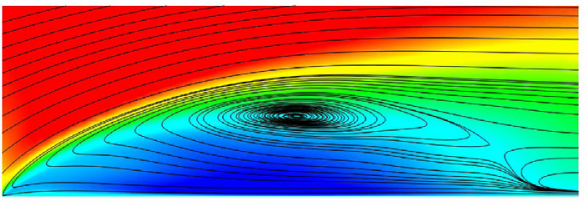

g)

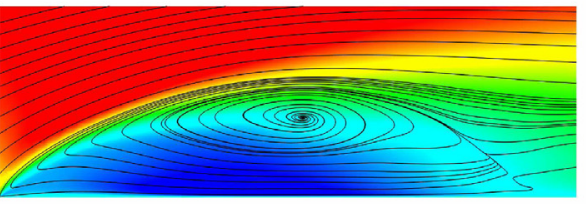

$\mathrm{u} / \mathrm{U}$

\begin{tabular}{rrrrr}
\hline-0.40 & -0.05 & 0.30 & 0.65 & 1.00
\end{tabular}

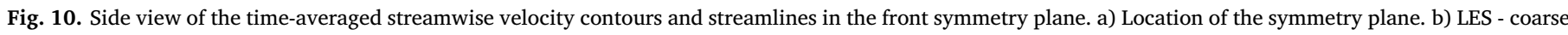

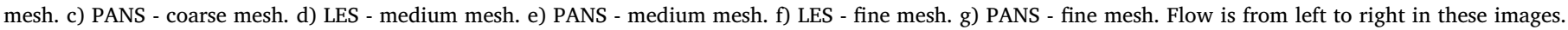

than in LES. In fact, the differences of the unresolved turbulence kinetic energy $k_{u}$ between PANS and LES are easily observable. For the same grid, a larger amount of $k_{u}$ is observed for PANS, indicating that it models more turbulence kinetic energy than LES. With the grid refinement, the region of $k_{u}$ is reduced, and more turbulence is resolved. Additionally, more information on the grid resolution can be obtained from the instantaneous input $f_{k}$, defined in Eq. (12), and the output $f_{k}$ (the ratio of unresolved to total turbulent kinetic energy), as shown in Fig. 14. Fig. 14 shows the influence of grid refinement on the instantaneous $f_{k}$ in PANS simulations.

Based on these $f_{k}$ contours, it is clear to see the level the flow structures that are resolved or modeled. For all cases, the $f_{k}$ values are high in the upstream and around the bow of the ship, and are low around the ship body and in the wake. High values mean that the flow is modeled rather than resolved. Note that the input $f_{k}$ is a conservative estimate as compared to output $f_{k}$. For the coarse mesh, the $f_{k}$ values in the symmetry plane is higher above the bridge and behind the funnel, as well as behind the two steps and in the wake, which corresponds to the contours of $k_{u}$. The fine grid resolves more turbulent kinetic energy, resulting in a lower value of $k_{u}$ as compared to the medium and the coarse grid, and consequently lower $f_{k}$ in the wake.

\subsection{Bi-stable behaviour of wake flow}

From the velocity profiles in Fig. 8 , an asymmetric velocity distribution can be observed. Here, it should be highlighted that the steady RANS simulation with $k-\varepsilon$ turbulence model fails to predict the flow asymmetry in the wake, as shown in Appendix A. This further justifies the use of unsteady simulations. To further understand this flow feature, Fig. 15 compares the time-averaged streamwise and instantaneous velocity contours and streamlines in the $z=0.22 \mathrm{~W}$ plane from LES and PANS. In general, using coarse grids, PANS and LES cannot predict the

a)
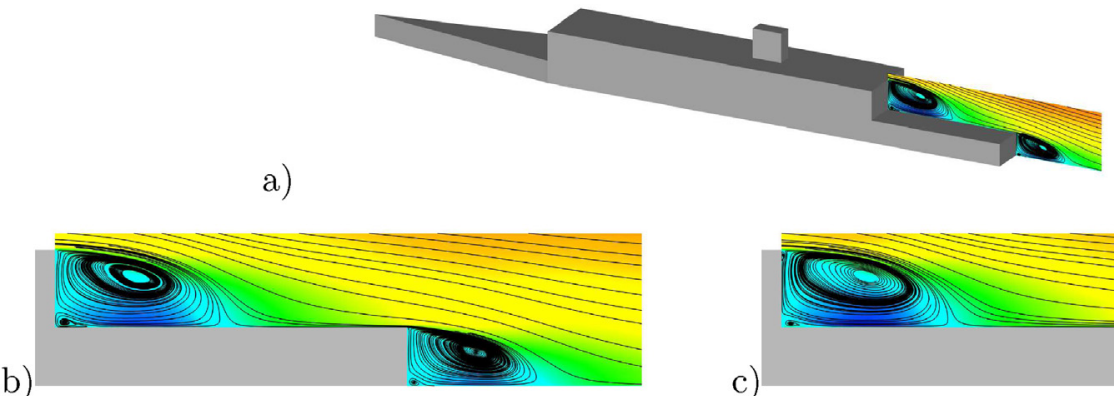

c)

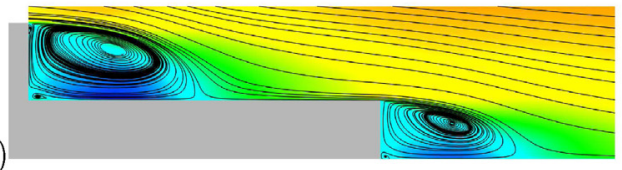

$\mathrm{u} / \mathrm{U}$

$\begin{array}{rrrrr}-0.40 & -0.05 & 0.30 & 0.65 & 1.00\end{array}$

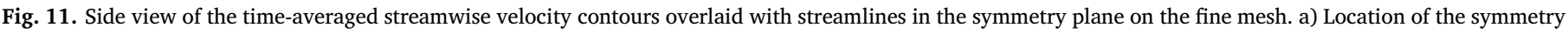
plane. b) LES. c) PANS. Flow is from left to right in these images. 
Table 3

Characteristics of the recirculation bubbles behind the hangar. $L(L=2 W)$ is the length of the flight deck and $h$ is the height of the hangar.

\begin{tabular}{|c|c|c|c|c|c|c|}
\hline Case & Cells (million) & $R e$ & $X_{R 1}(z / h=0.0667)$ & $X_{R 2}(z / h=0.0067)$ & $X_{V}$ & $Z_{V}$ \\
\hline LES & 5.87 & $8 \times 10^{4}$ & - & $0.577 L$ & $0.291 L$ & $0.682 h$ \\
\hline LES & 9.93 & $8 \times 10^{4}$ & $0.486 L$ & $0.535 L$ & $0.259 L$ & $0.652 h$ \\
\hline LES & 21.25 & $8 \times 10^{4}$ & $0.462 L$ & $0.504 L$ & $0.227 L$ & $0.658 h$ \\
\hline PANS & 5.87 & $8 \times 10^{4}$ & - & $0.532 L$ & $0.256 L$ & $0.657 h$ \\
\hline PANS & 9.93 & $8 \times 10^{4}$ & $0.463 L$ & $0.505 L$ & $0.239 L$ & $0.640 h$ \\
\hline PANS & 21.25 & $8 \times 10^{4}$ & $0.467 L$ & $0.502 L$ & $0.240 L$ & $0.654 h$ \\
\hline Exp. (Bardera and Meseguer, 2015) & - & $8 \times 10^{4}$ & $0.452 L$ & - & - & - \\
\hline DES (Forrest and Owen, 2010) & 5.8 & $2.26 \times 10^{7}$ & - & $0.490 L$ & - & - \\
\hline DES (Forrest and Owen, 2010) & 5.8 & $6.58 \times 10^{5}$ & - & $0.450 L$ & - & - \\
\hline
\end{tabular}

asymmetric flow characteristics behind the hangar, while the fine grid PANS and LES give better results, as shown by the velocity contour levels in these figures. This characteristic is more striking when the streamlines are added into the figure, (see Fig. 15). The mean flow is asymmetric and is characterized by two large vortices and some small size vortices. One large vortex is located on the upper side of the image whereas the other one is seen on the other side. With grid refinement, the upper one becomes narrower and more stretched (except for the medium grid LES simulation which shows an anti-symmetric flow state.), this flow configuration will be labeled $F C_{1}$. If we define an angle between the $y$ axis and the line passing through the centres of the two large vortices as $\theta$, the angle will increase with a higher resolution grid for the PANS simulations, while this angle remains a constant in the anti-symmetric flow states in LES. Note that in Fig. 15d the stretched vortex is seen on the $y<0$, henceforth referred to as $F C_{2}$. Therefore, it seems that this kind of flow is probably very unstable at the zero degree yaw angle and has a probability to result in either $F C_{1}$ or $F C_{2}$, bi-stable states. $F C_{1}$ is anti-symmetric to $F C_{2}$ with respect to the symmetry plane. Regarding the instantaneous flow on the fine mesh, Fig. 15h-i, a stagnant large vortex and a series of vortices are seen on the two opposite sides. This is consistent with the topology observed on the mean flow, with the larger vortex being observed on the same side.
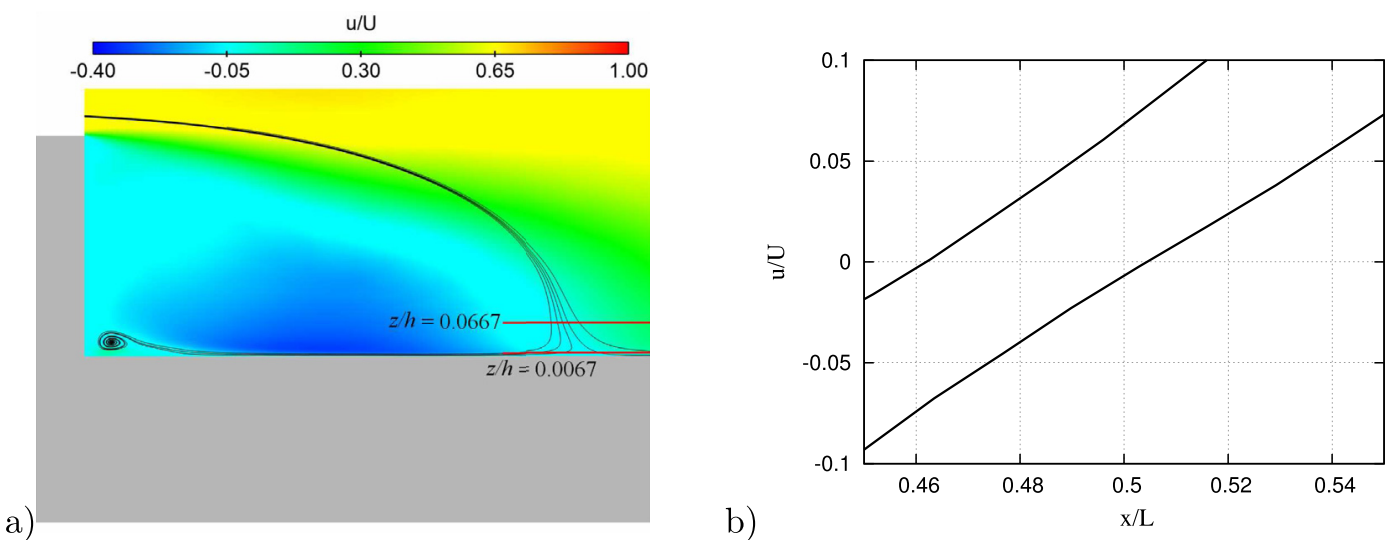

Fig. 12. Method for determining the reattachment length behind the first step. a) Time-averaged streamwise velocity contours and streamlines with two cutting lines. b) Velocity profiles along the lines. Flow is from left to right in image (a).
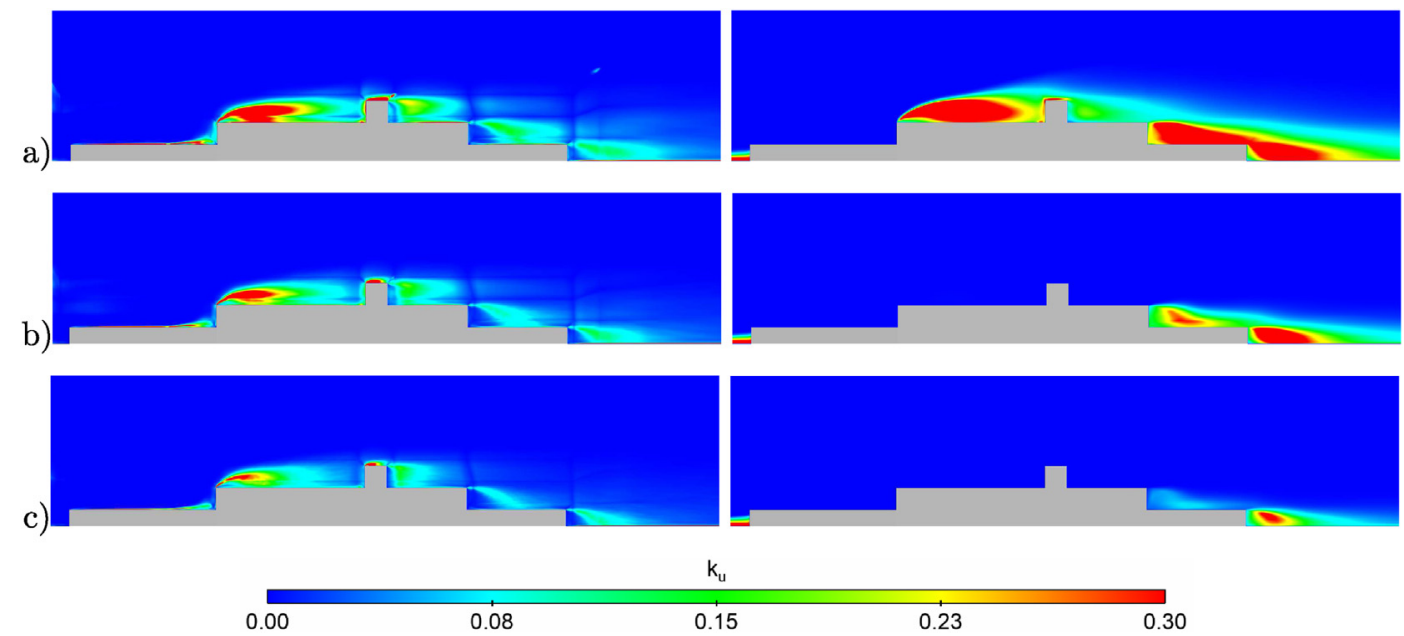

Fig. 13. Comparison of the unresolved turbulence kinetic energy. a) Coarse mesh. b) Medium mesh. c) Fine mesh. Left column: LES; right column: PANS. Flow is from left to right in these images. 


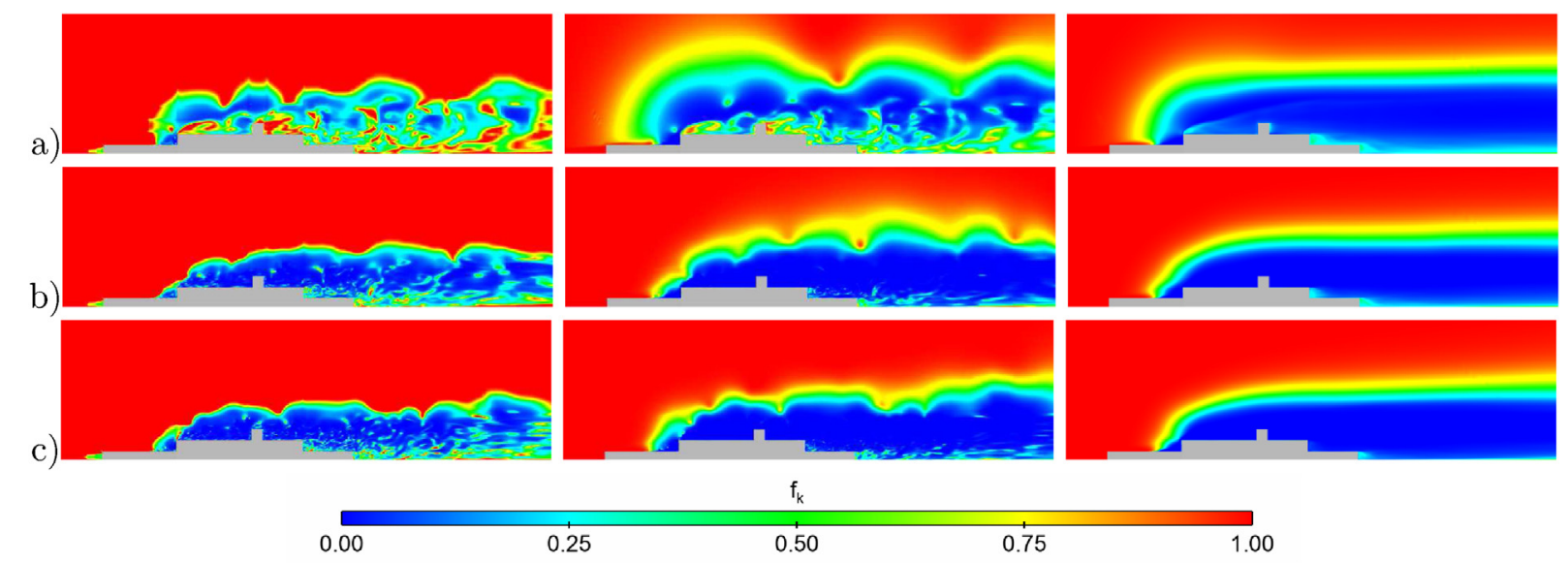

Fig. 14. Influence of grid refinement on the $f_{k}$ in PANS simulations: a) Coarse mesh; b) Medium mesh; c) Fine mesh. Left column: Input $f_{k}$; middle column: instantaneous output $f_{k}$; right column: time-averaged output $f_{k}$. Flow is from left to right in these images.

a)
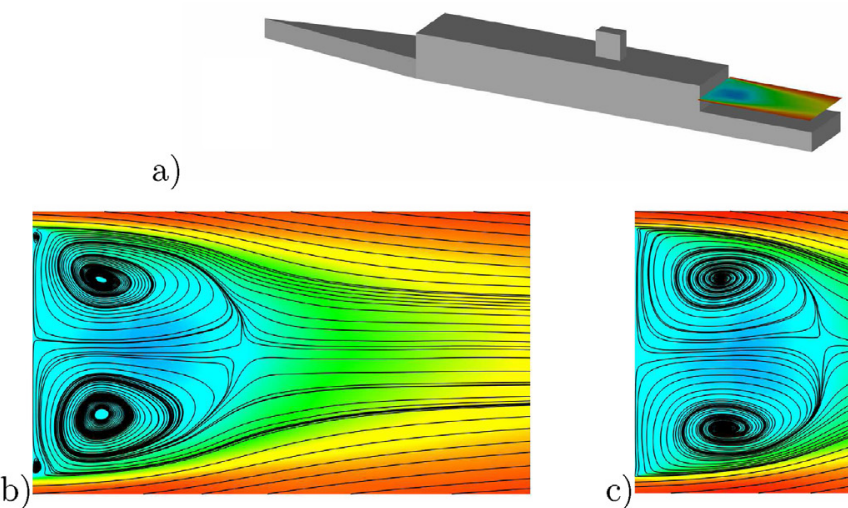

d)
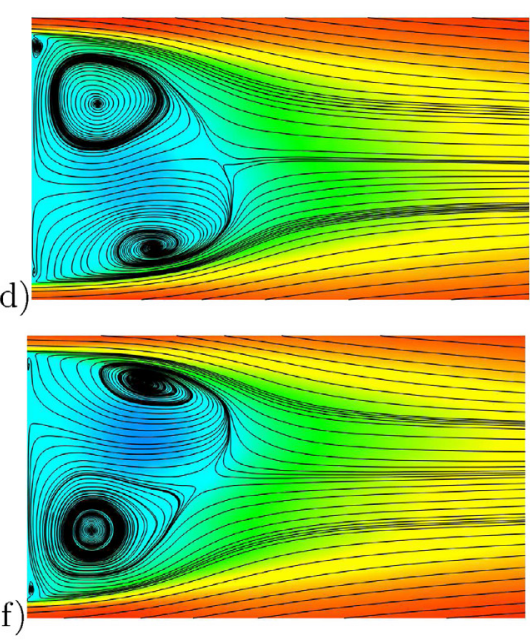

f)

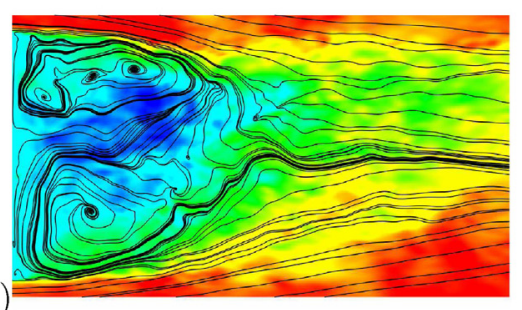

g)

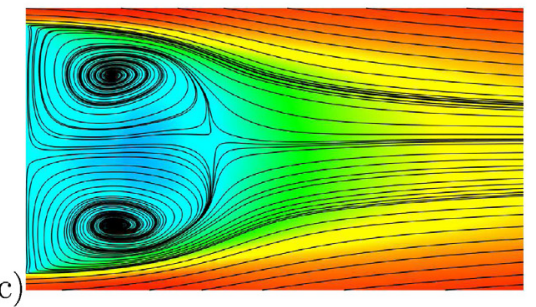

e)
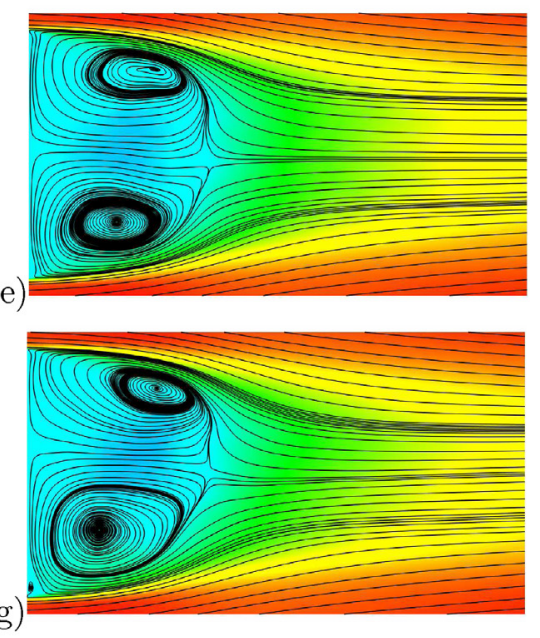

i)

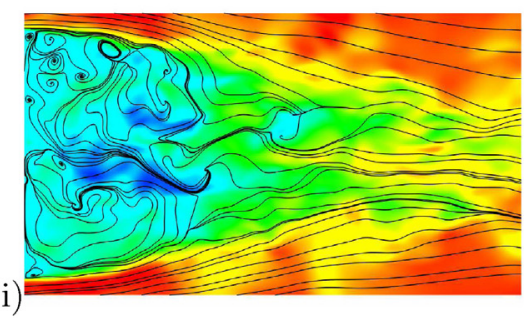

u/U

\begin{tabular}{rrrrr}
\hline 1 & -0.05 & 0.30 & 0.65 & 1.00
\end{tabular}

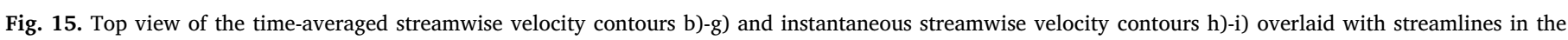

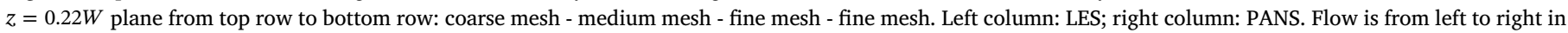
these images. 


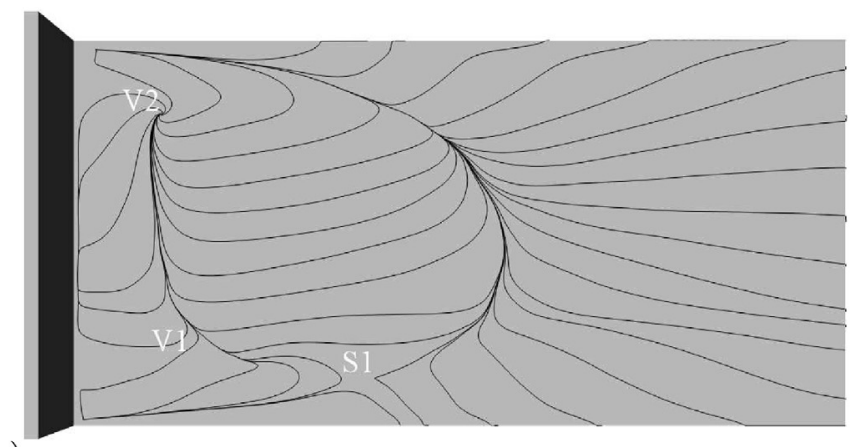

a)

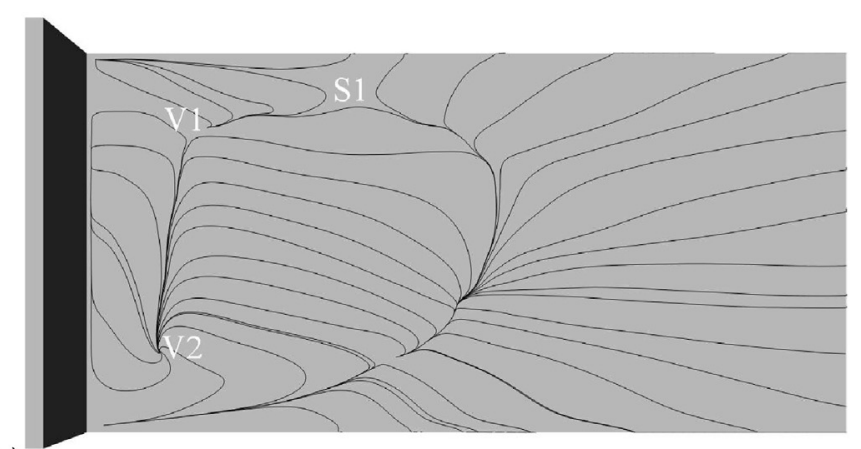

b)

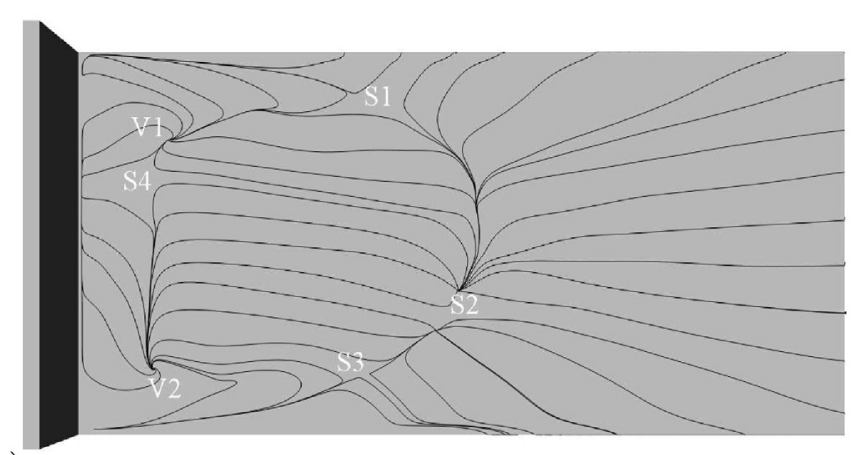

c)

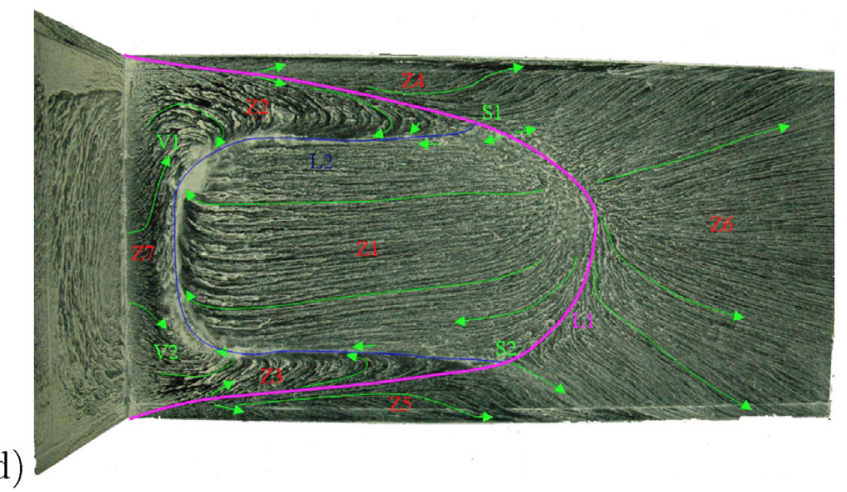

Fig. 16. Streamlines of the time-averaged velocity projected onto the flight deck of the ship. a) LES - medium mesh. b) LES - fine mesh. c) PANS - fine mesh. d) Oil flow visualization of a $\mathrm{SFSO}^{\prime}$ at $R_{W}=4.33 \times 10^{4}$, taken from Herry (2010).

A symmetric geometry with a zero degree yaw angle presents a far from expected symmetric velocity structure, which is also found in the flow around other similar bluff bodies (Herry, 2010). For example, tests focused on a SFSO' model which is similar to the SFS2 model were carried out in the TEMPO wind tunnel (Herry, 2010), show the same kind of asymmetric phenomenon in the flight deck, see Fig. 3.7 in Herry a)

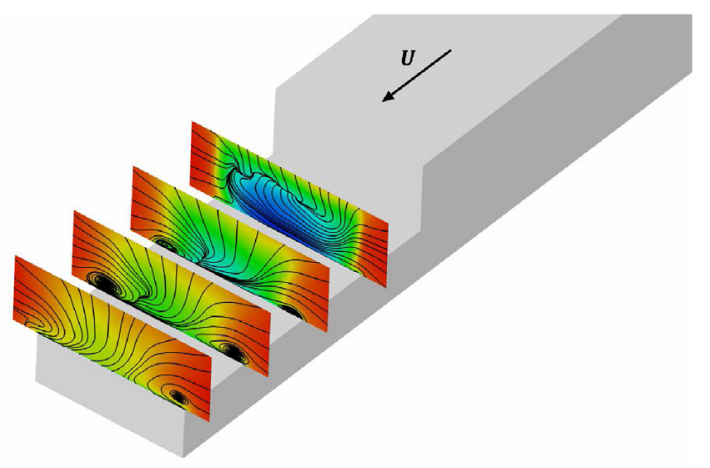

b)

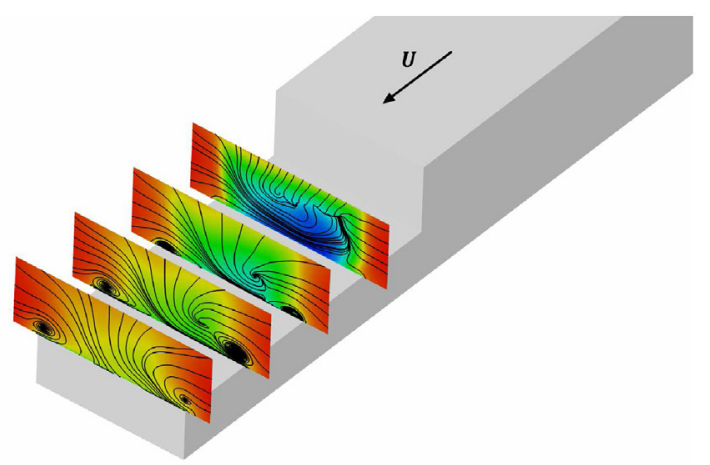

c)

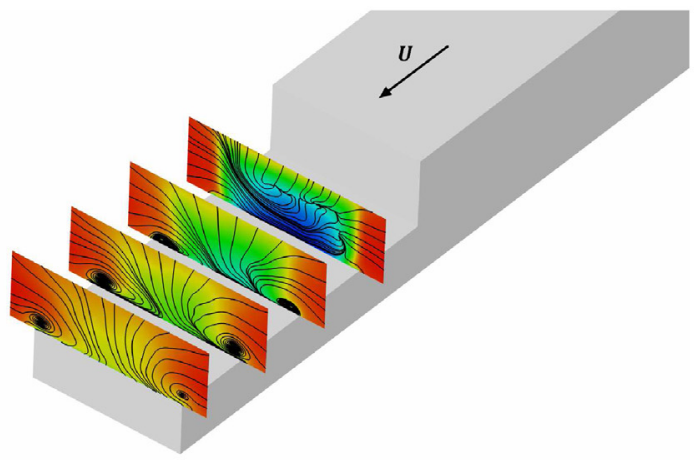

$\mathrm{u} / \mathrm{U}$

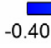

$-0.05$

0.30

1.00

Fig. 17. Velocity distributions in the planes at $25 \%, 50 \%, 75 \%$ and $100 \%$ length of the flight deck downstream of the hangar. a) LES - medium mesh. b) LES - fine mesh. c) PANS - fine mesh.

(2010). Where the SFSO' is based on the SFS1 model without funnel and with a pyramid nose in the front, Fig. 2.2 in Herry (2010). Although the geometry is slightly different from the model used in the present work, the bi-stable behaviour is prominent. Moreover, according to the test, it is confirmed that the bi-stability of flow is observed, and seems to be relatively independent of the upstream conditions.

To further explore this asymmetry, Fig. 16 shows the surface streamlines on the flight deck, and Fig. 17 shows the velocity distributions in four planes downstream the hangar, predicted by the medium and fine grid LES, and the fine grid PANS, respectively. The oil flow visualization of the SFSO' is used to make a comparison (this case is set at $-0.21^{\circ}$ yaw angle in the experiments). Overall, the flow patterns of simulations show similar results compared to those of wind tunnel tests, although the geometries used in both computations and tests are not exactly the same. According to the surface streamlines, the recirculation bubble behind the hangar can be observed as a nearparabolic shape. Within the recirculation bubble, backflow occurs towards the base of the hangar, and converges at the vortex V1 and vortex V2. In fact, in LES, vortex V2 is larger than vortex V1. The vortex center 
of V1 is difficult to locate, comparing to that for V2, since it is narrower and more stretched. It seems that vortex V2 is becoming the rotation center of the fluid very close to the flight deck, which indicates a slightly different behaviour compared to the streamlines illustrated in Fig. 15. Therefore, an asymmetry is clearly evident in Fig. 16 for LES. According to the singular points classification in Hunt et al. (1978), or called critical points in Perry and Chong (1987), there is one focus point V2 and one separation saddle point S1. The other critical points are not clearly visible, if they exist. On the other hand, in PANS, although the vortex V2 is slightly larger than vortex V1, the center of vortex V1 can be seen more clearly than the one in LES. Two foci V1 and V2 and four separation saddle points S1, S2, S3 and S4 are shown in the surface flow patterns, leading to a weaker asymmetry. This difference is also observed in the velocity distributions in the plane at $25 \%$ length of the flight deck downstream the hangar, Fig. 17. Note that in Fig. 17, the velocity, above the flight deck, close to the side of the stretched vortex V2 (see Fig. 16) is higher than the one on the other side. This behaviour is also shown in Syms (2008) using CFD and wind tunnel tests. Considering results from medium and fine grid LES, two anti-symmetric flow states are observed. In particular, the formation of the asymmetry inverts side, producing a specular flow field. The two flow states can be visualized in Fig. 17a and b, and the occurrence is very random, contributing to the change of velocity distributions in the wake. In this case, the occurrence of the two stable solutions is dependent on the spatial resolution, but a different mesh construction could have led to a similar result. The switch between the two flow states has previously been observed in the experiments (Grandemange et al., 2013). However, it is rather costly to simulate more than few seconds of the flow, and obtain sufficient running time to observe the switch in the asymmetry from the simulations.

\section{Conclusions}

The flow around a generic ship model was investigated to understand the flow physics at $R e=8 \times 10^{4}$. The model that consists of the bow, superstructure, bridge, hangar and flight deck is characterized by sharp edge separations on the top and side edges. Time-dependent simulations were performed using PANS and LES on three hexahedral grids of increasing resolution, as the steady RANS does not predict correct flow structures in the wake, as seen in Appendix A. The PANS results were compared with the corresponding LES and the available literature. To explore and assess the capability of PANS in predicting the unsteady turbulent flows, a comprehensive study of the resolved and modeled flow scales was carried out, including the time-averaged and the instantaneous flow structures around the ship, surface pressure coefficients, aerodynamic forces and unresolved turbulence kinetic energy. The results show that PANS simulations capture the main flow features and produce similar trends to those predicted by LES and the available experimental data. The coarse grid resolution in PANS leads it to act as URANS, resulting in an incorrect flow prediction in the separated regions. By refining the grid resolution, PANS captures more small-scale instantaneous flow structures, and the predictions are closer to LES. Large separation bubbles above the top of the bridge and in the wake are well-predicted by both PANS and LES. The time-averaged velocity profiles, contours and streamlines in the heli-deck show an asymmetric flow topology in the lateral plane, even though the geometry is aligned to the incoming flow. This flow phenomenon has already been investigated in Syms (2008) on the SFS2 model, and in Herry (2010) on the SFSO' model, and can be termed as flow bi-stability. Bi-stability has also been observed in other squareback bluff body flows such as the Ahmed body (Grandemange et al., 2013, 2015; Volpe et al., 2015) and ground transportation system (GTS) model (Krajnović et al., 2018; Schmidt et al., 2018). Moreover, this flow switches randomly from one state to the other at the zero degree yaw angle, contributing to the asymmetric flow distribution in the wake behind a squareback step (Herry, 2010; Herry et al., 2011). The mean drag coefficient $\left\langle C_{x}\right\rangle$ is around 0.70 for all cases, indicating that the bi-stable behaviour has little influence on the drag force coefficient. To conclude, the current study provides a baseline to incorporate flow control at the rear of the ship, where bi-stable flow occurs. Future studies will be aimed towards the use of flow control to achieve drag reduction and to control the bi-stable behaviour.

\section{Acknowledgements}

This work is supported financially by the Area of Advance Energy at Chalmers and the Swedish Energy Agency (Grant No. 43198-1).The authors are grateful to AVL List GmbH for providing the licenses for the AVL FIRE solver, and National Research Council of Canada for providing the experimental data of SFS2. The authors also acknowledge the computation time provided by SNIC (Swedish National Infrastructure for Computing) at the Center for Scientific Computing at Chalmers (C3SE) and the National Supercomputer Center (NSC) at Linköping University.

\section{Appendix A}

Here, a PANS simulation on the medium mesh using central differencing scheme (CDS) for the convective terms of the momentum equation was undetaken. The flow configuration $1\left(F C_{1}\right)$ is observed in the flight deck, where a large vortex is located on the lower side, with the other narrower and stretched vortex is seen on the upper side, as seen in Fig. 18a. However, the $\left\langle C_{x}\right\rangle$ and $\left\langle C_{z}\right\rangle$ are 0.6954 and 0.6918 with differences of approximately $1.5 \%$ and $6.7 \%$ lower, respectively, compared to those of PANS with AVL SMART Relaxed scheme in the same grid (see Table 2). While the CDS is generally more accurate, and requires meshes of higher resolution, AVL SMART schemes are slightly more diffusive, but lead to better convergence of the solutions. Thus, based on the results herein, AVL SMART schemes are more favorable for the present PANS simulations, as

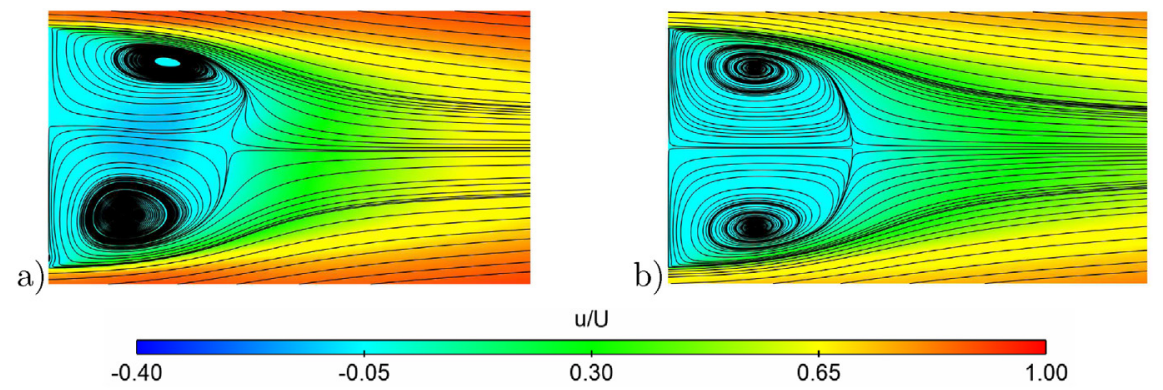

Fig. 18. Flow topology in the wake of the ship: a) On the medium mesh using PANS with CDS, b) On the fine mesh using RANS. Flow is from left to right in these images. Images are captured at $\mathrm{Z} / \mathrm{W}=0.22$. 
reported in previous work (Krajnović et al., 2016, 2018).

Additionally, the flow topology of the flight deck in the wake of the ship using steady $k-\varepsilon$ RANS turbulence model is shown in Fig. 18b. The simulation was carried out using the commercial finite volume CFD solver, AVL FIRE (AVL, 2014). The schemes used for convective terms and diffusive terms are the same as those in PANS. The normalized residual values of all quantities in the equations were set to $10^{-6}$. The steady RANS shows a symmetric flow structure in the wake on the fine mesh, failing to capture the asymmetric flow topology observed in Syms (2008). Thus, time-dependent methods, e.g., LES and PANS, were used in this study.

\section{Appendix B}

Here, Fig. 19 shows the time histories of the side forces and the PSD predicted by PANS and LES on the fine grid. The magnitudes of oscillations in the side forces are found to be almost the same in PANS and LES (Fig. 19a). Fig. 19b shows that the spectrum of the side force has several dominant peaks for non-dimensional frequencies up to $S t_{w}=0.5$, and the dominant $S t_{w}$ is around 0.2 .
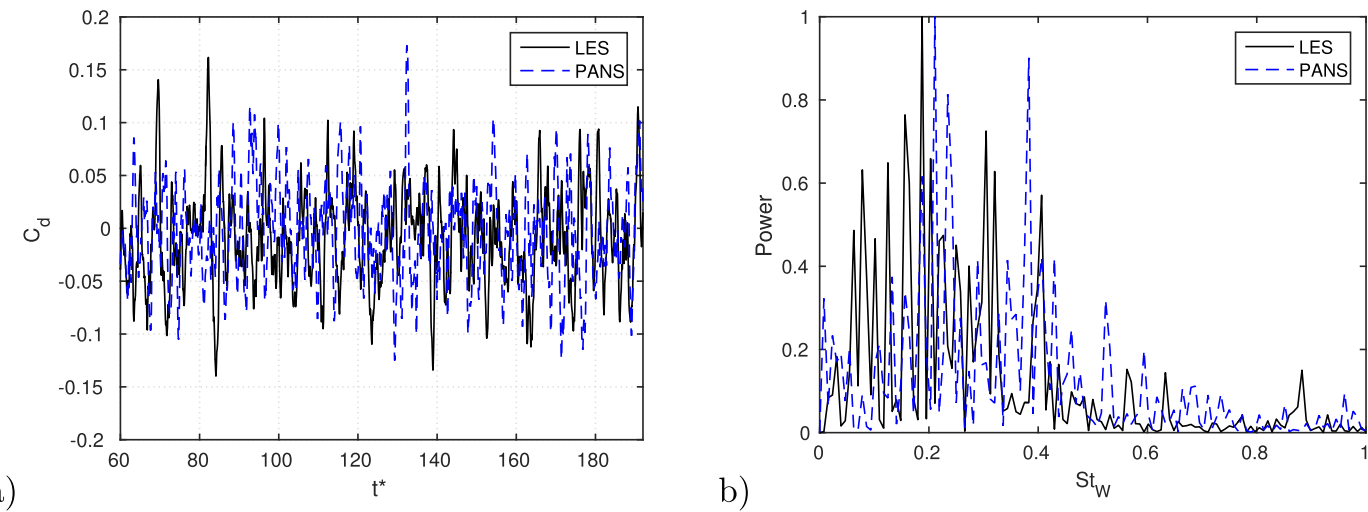

Fig. 19. a) Time histories of the side forces predicted by PANS and LES on the fine grid and b) the corresponding PSD.

\section{References}

AVL, 2014. AVL Fire Manual, V2014.

Bardera, R., Meseguer, J., 2015. Flow in the near air wake of a modified frigate, Proceedings of the Institution of Mechanical Engineers. Parting Gifts: J. Aero. Eng. 229, 1003-1012.

Basara, B., Krajnović, S., Girimaji, S., 2010. PANS Methodology Applied to Elliptic-relaxation Based Eddy Viscosity Transport Model. Springer, Berlin, Heidelberg, pp. 63-69. https://doi.org/10.1007/978-3-642-14139-3_7. https://doi.org/10.1007/ 978-3-642-14139-3 7

Basara, B., Krajnović, S., Girimaji, S., Pavlovic, Z., 2011. Near-wall formulation of the Partially Averaged Navier-Stokes turbulence model. AIAA J. 49, 2627-2636.

Forrest, J.S., Owen, I., 2010. An investigation of ship airwakes using Detached-Eddy Simulation. Comput. Fluids 39, 656-673.

Forrest, J., Kaaria, C., Owen, I., 2016. Evaluating ship superstructure aerodynamics for maritime helicopter operations through CFD and flight simulation. Aeronaut. J. 120, $1578-1603$.

Girimaji, S., 2006. Partially-Averaged Navier-Stokes model for turbulence: a ReynoldsAveraged Navier-Stokes to Direct Numerical Simulation bridging method. J. Appl. Mech. 73, 413-421.

Girimaji, S., Abdol-Hamid, K., 2005. Partially Averaged Navier-Stokes model for turbulence: implementation and validation. In: 43rd AIAA Aerospace Sciences Meeting and Exhibit, 10-13 January 2005, Reno, Nevada, pp. 502.

Girimaji, S., Srinivasan, R., Jeong, E., 2003. PANS turbulence model for seamless transition between RANS and LES: fixed-point analysis and preliminary results. In: ASME. Fluids Engineering Division Summer Meeting, Volume 2: Symposia, Parts A, B, and C, July 6-10, 2003, Hawaii, USA, pp. 1901-1909. https://doi.org/10.1115/ FEDSM2003-45336.

Girimaji, S., Jeong, E., Srinivasan, R., 2006. Partially Averaged Navier-Stokes method for turbulence: fixed point analysis and comparison with unsteady Partially Averaged Navier-Stokes. J. Appl. Mech. 73, 422-429.

Grandemange, M., Gohlke, M., Cadot, O., 2013. Bi-stability in the turbulent wake past parallelepiped bodies with various aspect ratios and wall effects. Phys. Fluids 25, 095103.

Grandemange, M., Cadot, O., Courbois, A., Herbert, V., Ricot, D., Ruiz, T., Vigneron, R. 2015. A study of wake effects on the drag of Ahmed's squareback model at the industrial scale. J. Wind Eng. Ind. Aerod. 145, 282-291.

Guilmineau, E., Deng, G., Wackers, J., 2011. Numerical simulation with a DES approach for automotive flows. J. Fluid Struct. 27, 807-816 IUTAM Symposium on Bluff Body Wakes and Vortex-Induced Vibrations (BBVIV-6).

Han, X., Krajnović, S., Basara, B., 2013. Study of active flow control for a simplified vehicle model using the PANS method. Int. J. Heat Fluid Flow 42, 139-150.

Hemida, H., Krajnović, S., 2009. Transient simulation of the aerodynamic response of a double-deck bus in gusty winds, ASME. J. Fluid Eng. 131, 031101-1-031101-10.

Hemida, H., Krajnović, S., 2010. LES study of the influence of the nose shape and yaw angles on flow structures around trains. J. Wind Eng. Ind. Aerod. 98, 34-46.

Herry, B., 2010. Aerodynamic study of a 3D Backward Facing Double Step Applied to Safer Launch and Recovery of Helicopters on Ships. Theses. Université de Valenciennes et du Hainaut-Cambresis. https://tel.archives-ouvertes.fr/tel00650410 .

Herry, B., Keirsbulck, L., Labraga, L., Paquet, J., 2011. Flow bistability downstream of three-dimensional double backward facing steps at zero-degree sideslip, ASME. Journal of Fluids Engineeing 133, 054501-1-054501-4.

Hu, G., Tse, K., Kwok, K., Zhang, Y., 2015. Large eddy simulation of flow around an inclined finite square cylinder. J. Wind Eng. Ind. Aerod. 146, 172-184.

Hunt, J.C.R., Abell, C.J., Peterka, J.A., Woo, H., 1978. Kinematical studies of the flows around free or surface-mounted obstacles; applying topology to flow visualization. J. Fluid Mech. 86, 179200.

Hunt, J.C.R., Wray, A.A., Moin, P., 1988. Eddies, streams, and convergence zones in turbulent flows. In: Studying Turbulence Using Numerical Simulation Databases, 2: Proceedings of the Summer Program, pp. 193-207.

Jeong, E., Girimaji, S., 2010. Partially Averaged Navier-Stokes (PANS) method for turbulence simulations-flow past a square cylinder. ASME. J. Fluid Eng. 132, 1212031-121203-11.

Kääriä, C.H., Wang, Y., White, M.D., Owen, I., 2013. An experimental technique for evaluating the aerodynamic impact of ship superstructures on helicopter operations. Ocean Eng. 61, 97-108.

Krajnović, S., 2009. Large eddy simulation of flows around ground vehicles and other bluff bodies, Philosophical Transactions of the Royal Society of London A: Mathematical. Physical and Engineering Sciences 367, 2917-2930.

Krajnović, S., Davidson, L., 2004. Exploring the flow around a simplified bus with large eddy simulation and topological tools. In: McCallen, R., Browand, F., Ross, J. (Eds.), The Aerodynamics of Heavy Vehicles: Trucks, Buses, and Trains. Springer Berlin Heidelberg, Berlin, Heidelberg, pp. 49-64.

Krajnović, S., Ringqvist, P., Basara, B., 2012a. Comparison of Partially Averaged NavierStokes and Large-Eddy Simulations of the flow around a cuboid influenced by crosswind, ASME. J. Fluid Eng. 134, 101202-1-101202-10.

Krajnović, S., Lárusson, R., Basara, B., 2012b. Superiority of PANS compared to LES in predicting a rudimentary landing gear flow with affordable meshes. Int. J. Heat Fluid Flow 37, 109-122.

Krajnović, S., Minelli, G., Basara, B., 2016. Partially-averaged Navier-Stokes simulations of two bluff body flows. Appl. Math. Comput. 272, 692-706.

Krajnović, S., Rao, A.N., Minelli, G., Zhang, J., Basara, B., 2018. Partially-Averaged Navier- Stokes simulations in engineering flows. In: 3rd Thermal and Fluids Engineering Conference (TFEC), Fort Lauderdale, FL, USA, March 4-7, 2018.

Kulkarni, P., Singh, S., Seshadri, V., 2007. Parametric studies of exhaust smoke-superstructure interaction on a naval ship using CFD. Comput. Fluids 36, 794-816. 
Lakshmipathy, S., Girimaji, S., 2010. Partially Averaged Navier-Stokes (PANS) method for turbulence simulations: flow past a circular cylinder, ASME. J. Fluid Eng. 132 121202-1-121202-9.

Luo, D., Yan, C., Liu, H., Zhao, R., 2014. Comparative assessment of PANS and DES for simulation of flow past a circular cylinder. J. Wind Eng. Ind. Aerod. 134, 65-77.

Minelli, G., Krajnović, S., Basara, B., Noack, B.R., 2016. Numerical investigation of active flow control around a generic truck a-pillar, Flow. Turbulence and Combustion 97 1235-1254.

Mirzaei, M., Krajnović, S., Basara, B., 2015. Partially-Averaged Navier-Stokes simulations of flows around two different Ahmed bodies. Comput. Fluids 117, 273-286.

Östh, J., Krajnović, S., 2014. A study of the aerodynamics of a generic container freight wagon using Large-Eddy Simulation. J. Fluid Struct. 44, 31-51.

Park, S., Yang, J., Rhee, S.H., 2017. Parametric study on ships exhaust-gas behavior using computational fluid dynamics. Engineering Applications of Computational Fluid Mechanics 11, 159-171.

Patankar, S., Spalding, D., 1972. A calculation procedure for heat, mass and momentum transfer in three-dimensional parabolic flows. Int. J. Heat Mass Tran. 15, 1787-1806.

Pereira, F., Vaz, G., Ea, L., 2015. On the numerical requirements of RANS and hybrid turbulence models. In: VI International Conference on Computational Methods in Marine Engineering (MARINE2015), Rome, Italy.

Pereira, F., Ea, L., Vaz, G., 2017. Verification and Validation exercises for the flow around the KVLCC2 tanker at model and full-scale Reynolds numbers. Ocean Eng. 129, $133-148$.

Pereira, F., Vaz, G., EÃßa, L., Girimaji, S., 2018. Simulation of the flow around a circular cylinder at $\mathrm{Re}=3900$ with Partially-Averaged Navier-Stokes equations. Int. J. Heat Fluid Flow 69, 234-246.

Perry, A.E., Chong, M.S., 1987. A description of eddying motions and flow patterns using critical-point concepts. Annu. Rev. Fluid Mech. 19, 125-155.

Piomelli, U., Chasnov, J., 1996. Large-eddy Simulations: Theory and Applications. Kluwer Academic Publisher.
Pope, S.B., 2001. Turbulent Flows. Cambridge University Press.

Pržulj, V., Basara, B., 2001. Bounded convection schemes for unstructured grids. In: 15th AIAA Computational Fluid Dynamics Conference, Fluid Dynamics and Co-located Conferences, 2593. American Institute of Aeronautics and Astronautics. https://doi. org/10.2514/6.2001-2593. https://doi.org/10.2514/6.2001-2593.

Rahimpour, M., Oshkai, P., 2016. Experimental investigation of airflow over the helicopter platform of a polar icebreaker. Ocean Eng. 121, 98-111.

Rao, A., Minelli, G., Basara, B., Krajnović, S., 2018. On the two flow states in the wake of a hatchback Ahmed body. J. Wind Eng. Ind. Aerod. 173, 262-278.

Schmidt, H.J., Woszidlo, R., Nayeri, C.N., Paschereit, C.O., 2018. The effect of flow control on the wake dynamics of a rectangular bluff body in ground proximity. Exp. Fluid 59, 107.

Smagorinsky, J., 1963. General circulation experiments with the primitive equations. Mon. Weather Rev. 91, 99-164.

Spalart, P., Jou, W., Strelets, M., Allmaras, S., 1997. Comments of feasibility of LES for wings, and on a hybrid RANS/LES approach. In: International Conference on DNS/ LES, Aug. 4-8, 1997, Ruston, Louisiana, pp. 137-147.

Syms, G., 2008. Simulation of simplified-frigate airwakes using a Lattice-Boltzmann method. J. Wind Eng. Ind. Aerod. 96, 1197-1206 5th International Colloquium on Bluff Body Aerodynamics and Applications.

Volpe, R., Devinant, P., Kourta, A., 2015. Experimental characterization of the unsteady natural wake of the full-scale square back Ahmed body: flow bi-stability and spectral analysis. Exp. Fluid 56, 99.

Zhang, J., Li, J., Tian, H., Gao, G., Sheridan, J., 2016. Impact of ground and wheel boundary conditions on numerical simulation of the high-speed train aerodynamic performance. J. Fluid Struct. 61, 249-261.

Zhang, J., Wang, J., Wang, Q., Xiong, X., Gao, G., 2018. A study of the influence of bogie cut outs' angles on the aerodynamic performance of a high-speed train. J. Wind Eng. Ind. Aerod. 175, 153-168. 